\title{
Pattern Formation during Interfacial Reaction in-between Liquid Sn and Cu Substrates - A Simulation Study
}

\author{
J. H. Ke ${ }^{\mathrm{a}, \mathrm{b}}$, Y. Gao ${ }^{\mathrm{a}}$, C. R. Kao ${ }^{\mathrm{b}}$, and Y. Wang ${ }^{\mathrm{a}}$ \\ ${ }^{a}$ Department of Materials Science \& Engineering, The Ohio State University, Columbus, Ohio 43210, USA

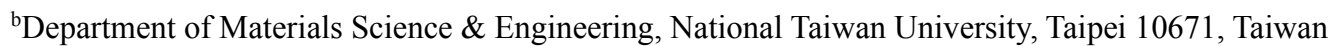

\begin{abstract}
Heteroepitaxial formation of $\eta-\mathrm{Cu}_{6} \mathrm{Sn}_{5}$ intermetallic compound (IMC) on unidirectional $\mathrm{Cu}$ has been found to exhibit remarkable microstructural features and provide opportunities for microstructure control of microelectronic interconnects, but the crystallographic origin and formation mechanism of such morphological patterns are still unclear. Here we investigate pattern formation during interfacial reactions in between liquid $\mathrm{Sn}$ and two types of unidirectional $\mathrm{Cu}$ substrates, (001) and (111), by using a combination of crystallographic analysis, theory of microelasticity and phase-field simulations. The simulation results show that each symmetry-related $\eta-\mathrm{Cu}_{6} \mathrm{Sn}_{5}$ variant grows along the direction of best atomic site matching with the substrates. No spatial alignment can be achieved when the growth takes place without heteroepitaxial constraints. In addition, anisotropy in interfacial energy between the liquid Sn and IMC is found to lead to faceted morphology. Further, the habit planes of $\eta-\mathrm{Cu}_{6} \mathrm{Sn}_{5}$ precipitates and kinematic compatibility across the interfaces are analyzed theoretically and found to be consistent with the simulations. These simulation predictions demonstrate excellent agreements with experimental observations.
\end{abstract}

Keywords: solder interconnect, intermetallic compound formation, heteroepitaxial growth, phase-field method, heteroepitaxial strain 


\section{INTRODUCTION}

Heteroepitaxial growth is an important technique to fabricate high-performance transistors and modern microelectronic interconnects. For traditional interconnects, chemical reactions between metallic substrates (such as $\mathrm{Cu}, \mathrm{Ag}$ or $\mathrm{Ni}$ ) and liquid solder alloys take place to form intermetallic compound (IMC) layers that establish metallurgical bonding between the solder and substrate [1]. Numerous studies have been carried out to investigate the formation, performance and reliability of IMCs from various perspectives, including phase stability and reactive diffusion kinetics [2-7], as well as microstructure evolution [8-12] and resistance to electric and mechanical loadings [13-19]. Recently, a promising way to enhance the properties of solder interconnects was developed by replacing the traditional polycrystalline $\mathrm{Cu}$ substrates with that of unidirectional $\mathrm{Cu}[20,21]$. The breakthrough opens up new opportunities for the development of novel microelectronic interconnects with significant reduction of structural defects and energy consumption.

Previous investigations $[10,22]$ have shown that a distinctive morphological difference arose when $\eta-\mathrm{Cu}_{6} \mathrm{Sn}_{5}$ formed on substrates of unidirectional or single crystal $\mathrm{Cu}$ as compared to that formed on polycrystalline $\mathrm{Cu}$. The IMCs on unidirectional $\mathrm{Cu}$ were found to have highly textured patterns with regular spatial arrangement. Furthermore, possible orientation relationships (ORs) between the $\eta-\mathrm{Cu}_{6} \mathrm{Sn}_{5}$ precipitates and the $\mathrm{Cu}$ substrates have been identified. Later similar phenomena were also found at the interface of $(\mathrm{Cu}, \mathrm{Ni})_{6} \mathrm{Sn}_{5} / \mathrm{Ni}[23], \mathrm{Ag}_{3} \mathrm{Sn} / \mathrm{Ag}$ [24] and $\mathrm{Cu}_{2}(\mathrm{In}, \mathrm{Sn}) / \mathrm{Cu}$ [25]. In most cases, IMCs on $\{001\}$ single crystal substrates grow preferentially along two perpendicular directions, whereas IMCs on $\{111\}$ substrates grow along three preferred directions with an $120^{\circ}$ angle in between. Especially, it was noted that all the preferred growth directions are the $<110>$ directions of the substrates. These unique features were rationalized by the argument that grains of the IMCs became elongated along these crystallographic directions because they have small atomic spacing mismatch between the IMCs and substrates [10]. Nevertheless, the explanation (based on one-dimensional (1D) atomic site mismatch) may not be valid for all instances because the interfacial 
pattern formation subjects to planar constraints in 2D. According to the edge-to-edge matching model [26], small atomic spacing mismatch along the close-packed direction between the matrix and IMC may indicate possible ORs that minimizes the interfacial energy. However, coherency elastic strain energy minimization plays a key role in controlling morphological pattern formation during epitaxial/heteroepitaxial growth [27, 28] as well as in solid state phase transformation [29] and should not be ignored.

In this paper, we employ a combination of crystallographic analysis, theory of microelasticity and phase-field simulations to study IMC growth in between liquid and substrate. Based on the minimization of the total free energy functional that includes chemical, interfacial and coherency elastic strain energies, the phase-field method accounts quantitatively for the heteroepitaxial strain arising from atomic site mismatch. Phase-field models that incorporate CALculation of PHAse Diagrams (CALPHAD) databases [30-32] have been utilized to study interfacial reactions in the $\mathrm{Cu}-\mathrm{Sn}$ solder system [33-35]. Nevertheless, most of the prior work focused on 2D morphological evolution and growth kinetics, and no attempt has been made to introduce the heteroepitaxial constraint into the model. More importantly, it is known that heteroepitaxial growth of one crystal on another crystalline substrate with higher symmetry will generate multiple orientation variants [36]. The appearance of multiple $\eta-\mathrm{Cu}_{6} \mathrm{Sn}_{5}$ variants (low symmetry) on unidirectional $\mathrm{Cu}$ substrates (high symmetry) have been identified by transmission electron microscopy (TEM) analysis [37], but the orientation relationship between the $\eta-\mathrm{Cu}_{6} \mathrm{Sn}_{5}$ and $\mathrm{Cu}$ substrate and the heteroepitaxial constraint from the substrate have never been connected to the experimentally observed morphological patterns of IMC and incorporated into simulations of $\mathrm{Cu}-\mathrm{Sn}$ interfacial reactions. To fully account for this effect and characterize the growth of different orientation variants of IMCs on crystalline substrates, it is highly desirable to integrate the available phase-field model of solder system with the microelasticity theory [29] that has been applied successfully to study coherent phase transformations in solids [38-41], epitaxial/heteroepitaxial film growth [42-49], dislocation/defect 
dynamics [50-52], as well as phase separation in $\mathrm{LiFePO}_{4}$ nanoparticles $[53,54]$.

In the present work, we focus on pattern formation during precipitation and heteroepitaxial growth of $\eta-\mathrm{Cu}_{6} \mathrm{Sn}_{5}$ on unidirectional (001) and (111) $\mathrm{Cu}$ substrates. We choose the $\mathrm{Cu}-\mathrm{Sn}$ system as the model system because it is one of the most studied and commonly-used combinations of materials for microelectronic interconnects. By taking full advantages of the microelasticity theory and phase-field approach, we simulate the pattern formation in both $2 \mathrm{D}$ and $3 \mathrm{D}$ in an attempt to provide insights into the strategies of developing IMC microstructures in a controllable manner. The paper is organized as the following. In Section 2 we present the crystallographic relation between $\eta-\mathrm{Cu}_{6} \mathrm{Sn}_{5}$ and two types of unidirectional $\mathrm{Cu}$ substrates, followed by symmetry analysis that gives quantitative descriptions of orientation variants and heteroepitaxial strain tensors. In Section 3, the construction of the phase-field model for the $\mathrm{Cu}-\mathrm{Sn}$ system is presented, including the chemical free energy, anisotropic interfacial energy, multiphase-field model, and the theory of microelasticity, followed by a general description of the simulation method. In Section 4 we present 2D and 3D phase-field simulation results showing the growth and evolution of $\eta-\mathrm{Cu}_{6} \mathrm{Sn}_{5}$ on unidirectional (001) and (111) $\mathrm{Cu}$ with different heteroepitaxial constraints. The simulation results are compared with experimental observations. In Section 5 we analyze the habit plane of each $\eta-\mathrm{Cu}_{6} \mathrm{Sn}_{5}$ variant and the condition of kinematic compatibility across the variant boundary. Furthermore, ORs predicted by the edge-to-edge matching model are compared with experimental studies. Limitations and possible extensions of the model are also addressed. Finally, a brief conclusion is given in Section 6.

\section{CRYSTALLOGRAPHIC ANALYSIS}

\subsection{Prediction of ORs between $\eta-C{ }_{6} \mathrm{Sn}_{5}$ and $\mathrm{Cu}$}

$\mathrm{Cu}$ possesses a cubic structure (space group: $F m \overline{3} m$ ) with $a_{\mathrm{Cu}}=3.615 \AA$ and $\eta-\mathrm{Cu}_{6} \mathrm{Sn}_{5}$ possesses a hexagonal structure (space group: $P 6_{3} / m m c$ ) with $a_{\eta}=4.190 \AA$ and $c_{\eta}=5.086 \AA$ [55], as illustrated in Figure 1. According to the edge-to-edge matching model [26], energy minimization of the interface 
between two phases can be achieved when close-packed (or relatively close-packed) rows of atoms and planes containing the close-packed rows are able to match across the interface. It can be shown from crystallographic analysis that the best matching close-packed row direction is $[0001]_{\eta} \|[110]_{\mathrm{Cu}}$ possessing an atomic mismatch of $0.51 \%$. The two best matching close-packed planes that contain the rows of atoms are $(1 \overline{2} 10)_{\eta} \|(1 \overline{1} 1)_{\mathrm{Cu}}$ and $(0 \overline{1} 10)_{\eta} \|(100)_{\mathrm{Cu}}$, each having a mismatch of interplanar space ( $d$-spacing) close to $0.38 \%$. The aforementioned matching direction and planes all suffice to the matching requirement of the edge-to-edge matching model. These ORs were mentioned with other possible ones identified by experimental work, including electron beam backscattered diffraction (EBSD), synchrotron x-ray microdiffraction, and TEM analysis $[9,10,37,56]$. One of the predicted ORs was also identified at the $(\mathrm{Cu}, \mathrm{Ni})_{6} \mathrm{Sn}_{5} /(001) \mathrm{Ni}$ interface in a TEM study [23]. Nevertheless, most of the previous work focused particularly on the atomic row matching without addressing the matching of planes (based on the $d$-spacing), which ensures that the set of planes can meet edge-to-edge at the interface and have only a small angle between them. The recent study [56] interpreted the experimental results by arbitrarily selecting the number of periodically matching atoms for finding possible minimal atomic mismatch. The interpretation leaded to significant underestimation of atomic mismatch along orthogonal directions and failed to realize the fact that if arbitrary selection of matching atoms can be done, then there will be no pattern formation observed in experiments. In the following crystallographic analysis of orientation variants and heteroepitaxial relations, the experimental results from Ref. [56] are utilized but we interpret them by following the edge-to-edge matching model [26].

\subsection{Orientation variants and heteroepitaxial strain of $\eta-C u_{6} \mathrm{Sn}_{5}$ on (001) $\mathrm{Cu}$}

According to the experimental results $[37,56]$, the $2 \mathrm{D}$ atomic site correspondence or heteroepitaxial relationship at the $\eta-\mathrm{Cu}_{6} \mathrm{Sn}_{5} /(001) \mathrm{Cu}$ interface was obtained as 


$$
\frac{3}{2}[110]_{(001) \mathrm{Cu}} \leftrightarrow[\overline{1} 100]_{(11 \overline{2} 0) \eta},[1 \overline{1} 0]_{(001) \mathrm{Cu}} \leftrightarrow[0001]_{(11 \overline{2} 0) \eta}
$$

where the subscript indicates the specific lattice plane. Figure 1(a) shows the schematic illustrations of atomic site matching at the interface $(001) \mathrm{Cu}$ and $(11 \overline{2} 0) \eta-\mathrm{Cu}_{6} \mathrm{Sn}_{5}$ according to the correspondence. Basically, an equivalent variant can be generated by a symmetry operation that is present in the substrate but absent in the product phase. By following the symmetry analysis $[36,57$, 58] at the lattice plane of contact interface, the number of equivalent orientation variants can be determined. Considering the $2 \mathrm{D}$ symmetry on a particular plane in a lattice, the $2 \mathrm{D}$ crystallographic point group of (001) $\mathrm{Cu}$ surface is $4 \mathrm{~mm}$ and that of $(11 \overline{2} 0) \eta-\mathrm{Cu}_{6} \mathrm{Sn}_{5}$ is $2 \mathrm{~mm}$. It can be demonstrated that the intersection group is $2 \mathrm{~mm}$ according to the heteroepitaxial relationship. The number of orientation variants arising from symmetry breaking is determined by $\mathrm{P}(4 \mathrm{~mm}) / \mathrm{P}(2 \mathrm{~mm})=2$, where $\mathrm{P}$ is the order of symmetry group. We note that the number of variants predicted by the symmetry analysis is in accord with the TEM result [37] showing a simultaneous appearance of two sets of $\eta-\mathrm{Cu}_{6} \mathrm{Sn}_{5}$ diffraction patterns when the zone axis was $[11 \overline{2} 0]_{\eta} \|[001]_{\mathrm{Cu}}$

The transformation matrix $\mathbf{F}$ can be obtained by the relation that associates the lattice vectors of the product phase with those of the substrate phase at the $\eta-\mathrm{Cu}_{6} \mathrm{Sn}_{5} /(001) \mathrm{Cu}$ interface. Here we consider the atomic site matching or heteroepitaxial constraint at the interface, instead of the lattice correspondence that is considered frequently in solid-state phase transformations in bulk. Such a determination of $\mathbf{F}$ has been utilized to describe the orientation and strain in heteroepitaxial growth [59], as well as epitaxial conditions of molecular organization on crystalline substrates $[60,61]$. It is calculated that when the reference coordinate $\mathrm{L}_{1}$ is linked to the cubic axes of the substrate lattice structure $\left(x_{1}\left\|[100]_{\mathrm{Cu}}, x_{2}\right\|[010]_{\mathrm{Cu}}\right)$, the correspondence can be described by considering a uniform deformation from substrate to product, and the transformation matrix of the first variant is given by

$$
\mathbf{F}_{1}=\left(\begin{array}{ll}
c_{\eta} / 2 \sqrt{2} a_{\mathrm{Cu}}+a_{\eta} / \sqrt{6} a_{\mathrm{Cu}} & c_{\eta} / 2 \sqrt{2} a_{\mathrm{Cu}}-a_{\eta} / \sqrt{6} a_{\mathrm{Cu}} \\
c_{\eta} / 2 \sqrt{2} a_{\mathrm{Cu}}-a_{\eta} / \sqrt{6} a_{\mathrm{Cu}} & c_{\eta} / 2 \sqrt{2} a_{\mathrm{Cu}}+a_{\eta} / \sqrt{6} a_{\mathrm{Cu}}
\end{array}\right)
$$


Generally, the transformation matrices of other variants can be determined by symmetry operations associated with the contact lattice planes. In this case the operation is just the change of sign of the off-diagonal components of $\mathbf{F}_{1}$. The stress-free epitaxial transformation strain tensor of each variant is calculated directly from the transformation matrix via Lagrange strain formula

$$
\boldsymbol{\varepsilon}^{00}(p)=\frac{1}{2}\left[\mathbf{F}_{p}^{T} \mathbf{F}_{p}-\mathbf{I}\right]
$$

where $p$ indicates the number of $\eta-\mathrm{Cu}_{6} \mathrm{Sn}_{5}$ variants, the superscript $T$ indicates transpose of the matrix, and $\mathbf{I}$ is the identity matrix. The two heteroepitaxial strain tensors are calculated as

$$
\boldsymbol{\varepsilon}^{00}(1)=\left(\begin{array}{cc}
-0.0287 & 0.0235 \\
0.0235 & -0.0287
\end{array}\right), \boldsymbol{\varepsilon}^{00}(2)=\left(\begin{array}{cc}
-0.0287 & -0.0235 \\
-0.0235 & -0.0287
\end{array}\right)
$$

Since $\eta-\mathrm{Cu}_{6} \mathrm{Sn}_{5}$ may possess different heteroepitaxial relationships with $\mathrm{Cu}$ substrates of different oriented directions, the results of crystallographic analysis in Section 2.2 should only be applicable for the heteroepitaxial growth on (001) $\mathrm{Cu}$.

\subsection{Orientation variants and heteroepitaxial strain of $\eta-C u 6 S_{5}$ on (111) Cu}

The orientation variants and their heteroepitaxial strain on (111) $\mathrm{Cu}$ can be obtained in a similar manner. The 2D heteroepitaxial relationship was shown by a recent TEM study [56] as

$$
[11 \overline{2}]_{(111) \mathrm{Cu}} \leftrightarrow[1 \overline{2} 10]_{(10 \overline{1} 0) \eta},[1 \overline{1} 0]_{(111) \mathrm{Cu}} \leftrightarrow[0001]_{(10 \overline{1} 0) \eta}
$$

Figure 1(b) shows the schematic illustrations of atomic site matching at the interface. It is noted that the first part of this heteroepitaxial relationship is different from the previous work [37], but here we adopt the recent result because it was analyzed based on the local cross-sectional microstructure showing the interfacial characteristic. The 2D crystallographic point group of the (111) Cu and (10T0) $\eta-\mathrm{Cu}_{6} \mathrm{Sn}_{5}$ planes are $6 \mathrm{~mm}$ and $2 \mathrm{~mm}$, respectively. Based on the identified relationship, the number of $\eta-\mathrm{Cu}_{6} \mathrm{Sn}_{5}$ variants that form on (111) $\mathrm{Cu}$ can be determined to be $\mathrm{P}(6 \mathrm{~mm}) / \mathrm{P}(2 \mathrm{~mm})=3$. The result is 
also consistent with the previous TEM analysis [37] which showed a simultaneous appearance of three sets of $\eta-\mathrm{Cu}_{6} \mathrm{Sn}_{5}$ diffraction patterns when the zone axis was $[11 \overline{2} 0]_{\eta} \|[\overline{1} 11]_{\mathrm{Cu}}$.

By choosing the reference coordinate $\mathrm{L}_{2}$ as $x_{1} \|[\overline{2} 11]_{\mathrm{Cu}}$ and $x_{2} \|[0 \overline{1} 1]_{\mathrm{Cu}}$ on the (111) $\mathrm{Cu}$ plane, the transformation matrix of the first variant is calculated as

$$
\mathbf{F}_{1}=\left(\begin{array}{cc}
\sqrt{6} a_{\eta} / 3 a_{\mathrm{Cu}} & 0 \\
0 & \sqrt{2} c_{\eta} / 3 a_{\mathrm{Cu}}
\end{array}\right)
$$

$\mathbf{F}_{2}$ and $\mathbf{F}_{3}$ can be determined by symmetry operations.

$$
\begin{aligned}
& \mathbf{F}_{2}=\left(\begin{array}{cc}
\sqrt{6} a_{\eta} / 12 a_{\mathrm{Cu}}+3 \sqrt{2} c_{\eta} / 8 a_{\mathrm{Cu}} & \sqrt{2} a_{\eta} / 4 a_{\mathrm{Cu}}-\sqrt{6} c_{\eta} / 8 a_{\mathrm{Cu}} \\
\sqrt{2} a_{\eta} / 4 a_{\mathrm{Cu}}-\sqrt{6} c_{\eta} / 8 a_{\mathrm{Cu}} & \sqrt{6} a_{\eta} / 4 a_{\mathrm{Cu}}+\sqrt{2} c_{\eta} / 8 a_{\mathrm{Cu}}
\end{array}\right) \\
& \mathbf{F}_{3}=\left(\begin{array}{cc}
\sqrt{6} a_{\eta} / 12 a_{\mathrm{Cu}}+3 \sqrt{2} c_{\eta} / 8 a_{\mathrm{Cu}} & \sqrt{6} c_{\eta} / 8 a_{\mathrm{Cu}}-\sqrt{2} a_{\eta} / 4 a_{\mathrm{Cu}} \\
\sqrt{6} c_{\eta} / 8 a_{\mathrm{Cu}}-\sqrt{2} a_{\eta} / 4 a_{\mathrm{Cu}} & \sqrt{6} a_{\eta} / 4 a_{\mathrm{Cu}}+\sqrt{2} c_{\eta} / 8 a_{\mathrm{Cu}}
\end{array}\right)
\end{aligned}
$$

The three heteroepitaxial strain tensors are then obtained.

$$
\boldsymbol{\varepsilon}^{00}(1)=\left(\begin{array}{cc}
-0.0522 & 0 \\
0 & -0.0051
\end{array}\right), \boldsymbol{\varepsilon}^{00}(2)=\left(\begin{array}{cc}
-0.0169 & 0.0204 \\
0.0204 & -0.0404
\end{array}\right), \boldsymbol{\varepsilon}^{00}(3)=\left(\begin{array}{cc}
-0.0169 & -0.0204 \\
-0.0204 & -0.0404
\end{array}\right)
$$

\section{MODEL}

We employ the multiphase-field model developed by Steinbach et al. [62, 63] to study the evolution of microstructures involving three phases, $\mathrm{Cu}(\alpha)$, liquid $\mathrm{Sn}(\mathrm{L})$, and $\eta-\mathrm{Cu}_{6} \mathrm{Sn}_{5}$ with multiple variants, whose structures are characterized by a set of non-conserved order parameters. The model has been applied not only to the fundamental studies of IMC nucleation and growth [34], but to more advanced processes such as transient liquid state bonding [64] and electromigration [35] as well. It should be noted that although both $\eta-\mathrm{Cu}_{6} \mathrm{Sn}_{5}$ and $\varepsilon-\mathrm{Cu}_{3} \mathrm{Sn}$ IMCs form during interfacial reactions according to the $\mathrm{Cu}$-Sn binary phase diagram and experiments, $\eta-\mathrm{Cu}_{6} \mathrm{Sn}_{5}$ has been shown to nucleate first at the early stage and possess a specific OR with the $\mathrm{Cu}$ substrate $[1,56]$. Therefore, the present study focuses on the formation of $\eta-\mathrm{Cu}_{6} \mathrm{Sn}_{5}$ and neglects that of $\varepsilon-\mathrm{Cu}_{3} \mathrm{Sn}$. The chemical free energy of 
each phase is formulated using the CALPHAD thermodynamic database for solder alloys. In particular, we introduce a two-sublattice model to calculate the chemical free energy of the stoichiometric compound $\left(\eta-\mathrm{Cu}_{6} \mathrm{Sn}_{5}\right)$. As determined by the bi-crystallography and symmetry argument, two orientation variants of $\eta-\mathrm{Cu}_{6} \mathrm{Sn}_{5}$ are generated on (001) $\mathrm{Cu}$ and three variants on (111) $\mathrm{Cu}$. Accordingly, we choose the correct order parameters to reflect the degeneracy of the orientation variants in our phase-field formulation. Those non-conserved order parameters are introduced in the model to characterize the spatial distribution of each orientation variant with prescribed stress-free heteroepitaxial strain, and the elastic strain energy is calculated by the microelasticity theory.

\subsection{Chemical free energy}

The chemical free energy density of $\mathrm{Cu}$ and liquid $\mathrm{Sn}$ are available in the binary CALPHAD database [65] and can be expressed as

$$
f_{\mathrm{ch}}^{\rho}=\sum_{i=\mathrm{Cu}, \mathrm{Sn}} x_{i}^{0} G_{i}^{\rho}+R T \sum_{i=\mathrm{Cu}, \mathrm{Sn}} x_{i} \ln x_{i}+{ }^{E} G^{\rho}
$$

where $x_{i}$ is the conserved order parameter or atomic fraction of species $i,{ }^{0} G_{i}^{\rho}$ is the Gibbs energy of the pure element $i$ in the chemical phase $\rho$. The last term corresponds to the excess Gibbs energy, which can be written by the Redlich-Kister polynomial:

$$
{ }^{E} G^{\rho}=x_{\mathrm{Cu}} x_{\mathrm{Sn}} \sum_{r=0}^{n}\left[{ }^{r} L_{\mathrm{Cu}, \mathrm{Sn}}^{\rho}\left(x_{\mathrm{Cu}}-x_{\mathrm{Sn}}\right)^{r}\right]
$$

where ${ }^{r} L_{\mathrm{Cu}, \mathrm{Sn}}^{\rho}$ is the temperature-dependent interaction parameters in the binary system. Because of the stoichiometric nature of $\eta-\mathrm{Cu}_{6} \mathrm{Sn}_{5}$, the chemical free energy was described as a temperature-dependent single value instead of a continuous function of composition [65]. The description causes a difficulty for any phase-field model because the variational derivative of free energy functional with respect to composition is not available. To resolve the issue, we follow the work of Moelans [66] by treating $\eta-\mathrm{Cu}_{6} \mathrm{Sn}_{5}$ as a chemical compound with small solubility and 
adopting a two-sublattice model $(\mathrm{Cu}, \mathrm{Sn})_{m}(\mathrm{Sn}, \mathrm{Cu})_{n}$. The chemical free energy of $\eta-\mathrm{Cu}_{6} \mathrm{Sn}_{5}$ is expressed based on the compound energy formulation:

$$
f_{\mathrm{ch}}^{\eta}=\sum_{i, j=\mathrm{Cu}, \mathrm{Sn}} y_{i}^{\mathrm{I}} y_{j}^{\mathrm{II} 0} G_{i: j}^{\eta}+R T\left(m \sum_{i=\mathrm{Cu}, \mathrm{Sn}} y_{i}^{\mathrm{I}} \ln y_{i}^{\mathrm{I}}+n \sum_{i=\mathrm{Cu}, \mathrm{Sn}} y_{i}^{\mathrm{II}} \ln y_{i}^{\mathrm{II}}\right)+{ }^{E} G^{\eta}
$$

where $y_{i}^{\mathrm{I}}$ and $y_{i}^{\mathrm{II}}$ are site fractions of species $i$ in sublattice I and II, respectively. ${ }^{0} G_{i: j}^{\eta}$ is the Gibbs energy of formation of the compound $i_{\mathrm{m}} j_{\mathrm{n}}$ or the so-called end member phases, and $m$ and $n$ give the ratio of sites on the two sublattice, which are 0.545 and 0.455 , respectively. The last term is the excess Gibbs energy, which was neglected in the study [66].

Figure 2 shows the Gibbs energy plots of $\mathrm{Cu}(\alpha)$, liquid $\mathrm{Sn}(\mathrm{L})$, and $\eta-\mathrm{Cu}_{6} \mathrm{Sn}_{5}$ chemical phases at $523 \mathrm{~K}$. The energy curve of $\eta-\mathrm{Cu}_{6} \mathrm{Sn}_{5}$ with large curvature at $x_{\mathrm{Cu}}=0.545$ clearly reflects its stoichiometric feature. Detailed thermodynamic parameters used in the present study are summarized in the Supplementary material (Table S1).

\subsection{Multiphase-field description}

The multiphase-field approach combined with the diffusion equation was applied in the studies of $\mathrm{Cu}-\mathrm{Sn}$ soldering reactions. Among them, Park and Arroyave [33] carried out extensive evaluation of parameters in the multiphase-field model of $\mathrm{Cu}-\mathrm{Sn}$ binary system. In the present study, a set of non-conserved order parameters $\phi_{\mathrm{i}}\left(i=1\right.$ to $\left.n_{p}\right)$ are introduced to describe the nucleation and growth of $\eta-\mathrm{Cu}_{6} \mathrm{Sn}_{5}$ at the liquid $\mathrm{Sn} / \mathrm{Cu}$ interface and characterize the evolution of each phase. $\phi_{i}=1\left(\phi_{j \neq i}=0\right)$ represents the existence of the phase $i . \phi_{1}$ and $\phi_{2}$ correspond respectively to the $\mathrm{Cu}$ and liquid $\mathrm{Sn}$ phases, while $\phi_{j}\left(j=3\right.$ to $\left.n_{p}\right)$ corresponds to the $(j-2)$ th orientation variant of $\eta-\mathrm{Cu}_{6} \mathrm{Sn}_{5}$. For $\eta-\mathrm{Cu}_{6} \mathrm{Sn}_{5}$ formed on (001) $\mathrm{Cu}$, two non-conserved order parameters are introduced to characterize the structural non-uniformities associated with the orientation variants, whereas for that on (111) $\mathrm{Cu}$, three non-conserved order parameters are introduced. The region in which non-conserved order parameters vary continuously between 0 and 1 corresponds to the interface or grain boundary. 
Following the multiphase-field description, a non-conserved order parameter can be taken as an interpolation function connecting the chemical free energy and phase composition of each chemical phase. The order parameters are constrained by $\sum_{i} \phi_{i}=1$. The total free energy functional is represented as

$$
F^{\mathrm{tot}}=\int_{V}\left\{\sum_{i, j(i<j)}\left[\frac{\kappa_{i j} a_{i j}^{2}}{2}\left(\phi_{j} \nabla \phi_{i}-\phi_{i} \nabla \phi_{j}\right)^{2}+\omega_{i j} \phi_{i} \phi_{j}\right]+\sum_{i} \phi_{i} \frac{f_{\mathrm{ch}}^{i}\left(x_{\mathrm{Cu}}^{i}\right)}{V_{m}}\right\} d V+F^{\text {elas }}
$$

where $\kappa_{i j}$ is the gradient energy coefficient of the interface between phases $i$ and $j, a_{i j}$ is an anisotropic function which will be defined in Section 3.4, $\omega_{i j}$ is the energy barrier of the double-well potential, $V_{m}$ is the molar volume, $F^{\text {elas }}$ is the elastic strain energy, which is calculated according to the theory of microelasticity and is presented in Section 3.3. $x_{\mathrm{Cu}}^{i}$ is the phase composition of $\mathrm{Cu}$ in the phase $i$, which is related to atomic fraction via $x_{\mathrm{Cu}}=\sum_{i} \phi_{i} x_{\mathrm{Cu}}^{i}$. The Kim-Kim-Suzuki (KKS) model [67] was employed in the phase-field model by considering the interface as a mixture of phases with equal chemical potentials. It is noted that the gradient energy term $\left(\phi_{j} \nabla \phi_{i}-\phi_{i} \nabla \phi_{j}\right)^{2}$ is different from some phase-field models of soldering reactions $[33,34]$. If we consider the interface or boundary between phase $i$ and $j$, the gradient energy term reduces to the general expression $\left(\nabla \phi_{i}\right)^{2}$. Mathematical details were presented by Steinbach et al. [62].

\subsection{Strain energy calculation}

The elastic strain energy functional is formulated by the microelasticity theory [29] :

$$
E^{\text {elas }}=\frac{1}{2} \int C_{i j k l} \varepsilon_{i j}^{*} \varepsilon_{k l}^{*} d V+\frac{V}{2} C_{i j k l} \bar{\varepsilon}_{i j} \bar{\varepsilon}_{k l}-\bar{\varepsilon}_{i j} \int C_{i j k l} \varepsilon_{k l}^{*} d V-\frac{1}{2} \int \frac{d^{3} g}{(2 \pi)^{3}} n_{i} \tilde{\sigma}_{i j}^{*} \Omega_{j k}^{0} \tilde{\sigma}_{k l}^{*} n_{l}
$$

where $C_{i j k l}$ is the elastic stiffness tensor, $\varepsilon_{i j}^{*}$ is the eigenstrain, which can be expressed as a linear combination of the order parameters of $\eta-\mathrm{Cu}_{6} \mathrm{Sn}_{5}$ variants and their stress-free heteroepitaxial strain tensors: 


$$
\varepsilon_{i j}^{*}=\sum_{k=3}^{n_{p}} \phi_{k} \varepsilon_{i j}^{00}(k-2)
$$

$\bar{\varepsilon}_{i j}$ is the prescribed macroscopic strain if the strain-controlled boundary condition is employed,

$\Omega_{i k}^{0-1}=C_{i j k l} n_{l} n_{j}, n_{i}=g_{i} /\left|g_{i}\right|$, and $g_{i}$ is a vector in the reciprocal space. $\tilde{\sigma}_{i j}^{*}=C_{i j k l} \tilde{\varepsilon}_{k l}^{*}$, where $\tilde{\varepsilon}_{k l}^{*}$ is the Fourier transform of $\varepsilon_{k l}^{*}$. The last integral in the reciprocal space excludes the point at $g=0$.

We note that because the effect of in-plane heteroepitaxial strain at the interface between $\mathrm{Cu}$ and $\eta-\mathrm{Cu}_{6} \mathrm{Sn}_{5}$ is known to be the dominant source of heteroepitaxial constraints affecting the pattern formation at the interface, the out-of-plane strain incompatibility is not taken into account by making the out-of-plane components of eigenstrain zero. In other words, the treatment excludes any elastic constraint along the direction normal to the interface.

\subsection{Anisotropic interfacial energy}

The $\eta-\mathrm{Cu}_{6} \mathrm{Sn}_{5}$ crystal in liquid $\mathrm{Sn}$ was found to exhibit a highly anisotropic shape and faceted interface with six-fold symmetry on the basal plane [1]. However, the $\gamma$-plot of $\eta-\mathrm{Cu}_{6} \mathrm{Sn}_{5}$ characterizing the hexagonal anisotropy of interfacial energy has not been evaluated and identified. To take the interfacial anisotropy into account in an approximated but quantitative manner, we follow the treatment developed by Wendler et al. [68] and introduce an anisotropic function $a_{i j}$ in a piecewise-defined form by using the maximum condition:

$$
a_{i j}=\max _{1 \leq k \leq n}\left\{\frac{\phi_{j} \nabla \phi_{i}-\phi_{i} \nabla \phi_{j}}{\left|\phi_{j} \nabla \phi_{i}-\phi_{i} \nabla \phi_{j}\right|} \cdot \vec{\eta}_{k, i j}\right\}
$$

where the first term in the braces characterizes the unit vector of interface normal, the second term denotes the set of vertex vectors for the corresponding Wulff construction, and $n$ indicates the number of defined vertex vectors. The interfacial energy is isotropic if the anisotropic function is a constant. We consider only the $\eta-\mathrm{Cu}_{6} \mathrm{Sn}_{5} / \mathrm{Sn}$ interface to be anisotropic and assume that the anisotropic function is equal to 1 for each other phase boundaries. For the interface between hexagonal 
$\eta-\mathrm{Cu}_{6} \mathrm{Sn}_{5}$ and $\mathrm{Sn}$, we define 12 vertex vectors by letting two of them be parallel to the $[1,1.732, \pm 1.5]$ directions (based on a Cartesian coordinate with the third component normal to the basal plane) while keeping the magnitude of the vectors equal to unity, and then six-fold rotations are applied about the [001] direction to obtain the other 10 vertex vectors. It is noted that a rotation matrix has to be imposed on each $\eta-\mathrm{Cu}_{6} \mathrm{Sn}_{5}$ variant to align the Cartesian coordinate with respect to the computational coordinate and let the $[0001]_{\eta}$ direction follow the crystallographic relation.

\subsection{Kinetic equations}

In the phase-field model, the time-evolution of non-conserved order parameters is governed by the time-dependent Ginzburg-Landau equation. Steinbach et al. [63] derived the governing equation for the multiphase-field theory as a superposition of pairwise interaction between phase $i$ and other coexisting phases. The pairwise interaction is weighted by the mobility of each pair of phases. The governing equation of non-conserved order parameters was derived as [63]

$$
\frac{\partial \phi_{i}}{\partial t}=-\frac{2}{N_{p}} \sum_{j(j \neq i)} s_{i} s_{j} a_{i j}^{2} L_{i j}\left(\frac{\delta F^{\mathrm{tot}}}{\delta \phi_{i}}-\frac{\delta F^{\mathrm{tot}}}{\delta \phi_{j}}\right)+\zeta(\mathbf{r}, t)
$$

where $N_{p}$ is the local number of coexisting phases, $s_{i}$ is a step function which is equal to 1 when the phase $i$ exists and is 0 otherwise, $L_{i j}$ is the mobility of the interface between phases $i$ and $j$, and $\zeta$ is the Langevin-noise terms describing thermal fluctuations of the order parameters [69]. The time-evolution of the conserved order parameter or atomic fraction is governed by the Cahn-Hilliard equation [70]:

$$
\frac{\partial x_{\mathrm{Cu}}}{\partial t}=V_{m}^{2} \nabla \cdot\left[\tilde{M} \nabla\left(\frac{\delta F^{\mathrm{tot}}}{\delta x_{\mathrm{Cu}}}\right)\right]
$$

The term in square brackets represents the negative atomic flux of $\mathrm{Cu}$ defined in the laboratory frame of reference and $\tilde{M}$ is the corresponding chemical mobility. 


\subsection{Description of simulations}

The model is first demonstrated by $2 \mathrm{D}$ simulations of $\eta-\mathrm{Cu}_{6} \mathrm{Sn}_{5}$ growth on (001) and (111) $\mathrm{Cu}$, followed by simulations in 3D. The $2 \mathrm{D}$ work was carried out by considering the nucleation and growth of $\eta-\mathrm{Cu}_{6} \mathrm{Sn}_{5}$ in $\mathrm{Cu}$-supersaturated liquid $\mathrm{Sn}$ on a unidirectional substrate which maintains continuous growth of $\eta-\mathrm{Cu}_{6} \mathrm{Sn}_{5}$. The 2D computational supercell was discretized into a system of 192 $\times 192$ grids. Initial nucleation was only allowed at the center of the computational supercell for local nucleation. The anisotropic interfacial energy was temporarily not considered in the 2D work for better demonstration of the effect of heteroepitaxial constrains, stress fields, and coherency elastic strain energy, which will be analyzed and quantified more precisely in Section 5 .

Figure 3 shows the schematic illustration of the initial 3D computational supercell in this study. All the energy contributions were considered in 3D simulations. The mesh spacing was $2 \mathrm{~nm}$ and the interfacial width consisted of 5 mesh grids (in both 2D and 3D work). The 3D computational supercell was discretized into a system of $128 \times 128 \times 48$ grids. Periodic boundary conditions were assumed along the $\mathrm{x}$ - and $\mathrm{y}$-directions, and a no-flux boundary condition was enforced explicitly at the bottom and top surfaces. Under the initial condition, liquid Sn and unidirectional (001) or (111) $\mathrm{Cu}$ contacted with each other at a planar interface without initial formation of $\eta-\mathrm{Cu}_{6} \mathrm{Sn}_{5}$. Langevin-noise terms describing thermal fluctuations of the order parameters were introduced in the last term of Eq. (15) during the initial stage in order to induce the nucleation of $\eta-\mathrm{Cu}_{6} \mathrm{Sn}_{5}$. In addition to the modeling of $\eta-\mathrm{Cu}_{6} \mathrm{Sn}_{5}$ formation on (001) and (111) $\mathrm{Cu}$, we also presents the simulation without considering heteroepitaxial constraint or elastic strain energy for comparison. It has been shown in the EBSD study by Kumar et al. [71] that no preferred orientation relationship was found between $\eta-\mathrm{Cu}_{6} \mathrm{Sn}_{5}$ and polycrystalline $\mathrm{Cu}$ at the interface. Therefore, the simulation without heteroepitaxial relationship can be regarded as an approximated case of IMC formation on traditional electroplating or polycrystalline $\mathrm{Cu}$ where no specific heteroepitaxial relationship with $\mathrm{Cu}$ can be 
achieved for each $\eta-\mathrm{Cu}_{6} \mathrm{Sn}_{5}$ grain. All the simulation results are compared with experiments of interfacial reactions.

The $\mathrm{Cu}$-substrate and $\eta-\mathrm{Cu}_{6} \mathrm{Sn}_{5}$-precipitate system was treated to be elastically homogeneous for simplicity. A stress-free boundary condition was employed along the z-direction, whereas a clamp or zero-strain boundary condition was prescribed along the $\mathrm{x}$ - and y-directions. Elastic properties of $\eta-\mathrm{Cu}_{6} \mathrm{Sn}_{5}$ were taken from the experimentally measured Young's modulus, shear modulus, and Poisson's ratio, which were $9.69 \times 10^{10} \mathrm{~N} \mathrm{~m}^{-2}, 3.70 \times 10^{10} \mathrm{~N} \mathrm{~m}^{-2}$ and 0.309 respectively [17].

We adopted the kinetic parameters assessed by Park and Arroyave [33] in the phase-field model of soldering reactions. Due to the lack of reliable experimental data, the interfacial energies of $\mathrm{Cu}(\alpha) / \eta-\mathrm{Cu}_{6} \mathrm{Sn}_{5}, \eta-\mathrm{Cu}_{6} \mathrm{Sn}_{5} / \mathrm{Sn}(\mathrm{L})$, and interphase boundaries (or grain boundaries) of $\eta-\mathrm{Cu}_{6} \mathrm{Sn}_{5}$ with respect to temperature and orientation are difficult to be determined and verified. For simplicity, we followed the previous work by assuming all the interfacial energies to be isotropic and constant, except that the $\eta-\mathrm{Cu}_{6} \mathrm{Sn}_{5} / \mathrm{Sn}$ interface was treated to be anisotropic by following Section 3.4. The interfacial energies of $\mathrm{Cu} / \eta-\mathrm{Cu}_{6} \mathrm{Sn}_{5}, \eta-\mathrm{Cu}_{6} \mathrm{Sn}_{5} / \mathrm{Sn}, \mathrm{Cu} / \mathrm{Sn}$ interfaces, and $\eta-\mathrm{Cu}_{6} \mathrm{Sn}_{5}$ interphase boundaries were considered to be $0.30,0.10,0.35$, and $0.30 \mathrm{~J} \mathrm{~m}^{-2}$, respectively. The widths of all the interfaces or interphase boundaries were assumed to be $10 \mathrm{~nm}$. It is noted that a relatively large interfacial width is required for the length scale considered in the current study. Because the key interfacial properties that control the kinetics of the microstructure evolution are the interfacial energy and mobility (diffusion-controlled growth has been ensured in the simulations) and they are independent of the interfacial width in the current model, the effect of boundary width on the kinetics can be ignored. According to the gradient thermodynamics, the gradient energy coefficient and energy barrier height in Eq. (11) can be obtained as

$$
\kappa_{i j}=\frac{8 w_{i j} \sigma_{i j}^{\mathrm{int}}}{\pi^{2}} \text { and } \omega_{i j}=\frac{4 \sigma_{i j}^{\mathrm{int}}}{w_{i j}}
$$


where $w_{i j}$ and $\sigma_{i j}^{\text {int }}$ are the width and interfacial energy of the interface between phases $i$ and $j$, respectively.

\section{RESULTS}

\section{$4.12 \mathrm{D}$ simulation of $\eta-\mathrm{Cu} 6 \mathrm{Sn} 5$ formation on (001) $\mathrm{Cu}$}

Figure 4(a) - (c) shows the time-evolution of the $\eta-\mathrm{Cu}_{6} \mathrm{Sn}_{5}$ variants (dash line) with the distributions of coherency stress field $\left(\sigma_{11}, \sigma_{22}\right.$, and $\left.\sigma_{11}+\sigma_{22}\right)$ on $(001) \mathrm{Cu}$. The calculations of stress on (001) plane of $\mathrm{Cu}$ was based on the $\mathrm{L}_{1}$ coordinate. The blue and orange dash lines correspond to the boundaries of variant 1 and variant 2 , respectively. Shortly after the nucleation of $\eta-\mathrm{Cu}_{6} \mathrm{Sn}_{5}$, tensile stress was generated around the center of the nucleus. Because of the negative trace of each heteroepitaxial strain tensor and the imposed clamp boundary condition, it is expected that tensile stress can be generated macroscopically by the nucleation and growth of $\eta-\mathrm{Cu}_{6} \mathrm{Sn} 5$. Nevertheless, slight local compression can be seen close to the tips of variants during the initial stage, while at the later stage the compressive stress gradually decreased with time. The tensile area was found to increase with the growth of $\eta-\mathrm{Cu}_{6} \mathrm{Sn}_{5}$, and the magnitude of tensile stress also increased. Particularly, the strong tensile stress was produced at the grooves of two variants, as shown by the plot of the invariant $\left(\sigma_{11}+\sigma_{22}\right)$ in Figure 4(c). It can be seen that local and concentrated compressive stress is generated at the inner tip of the boundary of two variants. The stress field distribution indicates that the grooves with high tensile stress will be elastically unfavorable for further growth of IMC or formation of multi-variant configurations. The new nucleation process will be more preferred to take place at the tips of variants.

The pattern in Figure 4 demonstrates pronounced preferred growth and elongation along two perpendicular directions. The preferred directions were not evident initially because of the strong chemical driving force of nucleation, but became more pronounced afterward as the growth of $\eta-\mathrm{Cu}_{6} \mathrm{Sn}_{5}$ increased the overall contribution of elastic strain energy. Based on the $\mathrm{L}_{1}$ coordinate, it can 
be shown that the two aligned directions perpendicular to each other belong to the $<110>$ direction of $\mathrm{Cu}$ and exhibit a windmill pattern. The 2D pattern formation is in good agreement with that characterized by SEM and EBSD analysis [72]. Most importantly, the simulation shows that the elongated directions of variant 1 and variant 2 correspond to the principal directions of the heteroepitaxial strain tensors (Eq. (3)) with minimal eigenvalues, which are [110] and [110] of $\mathrm{Cu}$, respectively. The minimal eigenvalue is $-0.51 \%$ and it reflects the minimum atomic mismatch between $\eta-\mathrm{Cu}_{6} \mathrm{Sn}_{5}$ and $\mathrm{Cu}$.

\section{$4.22 \mathrm{D}$ simulation of $\eta-\mathrm{Cu}_{6} \mathrm{Sn}_{5}$ formation on (111) $\mathrm{Cu}$}

Figure 5(a) - (c) shows the time-evolution of the $\eta-\mathrm{Cu}_{6} \mathrm{Sn}_{5}$ variants (dash line) accompanied with the distributions of coherency stress field $\left(\sigma_{11}, \sigma_{22}\right.$, and $\left.\sigma_{11}+\sigma_{22}\right)$ on $(111) \mathrm{Cu}$ based on the $\mathrm{L}_{2}$ coordinate. The blue, orange, and red dash lines correspond to the boundaries of variant 1 , variant 2 , and variant 3, respectively. After initial nucleation, tensile stress was produced around the nucleus, but compressive stress was also generated at the center. The nucleation and growth of $\eta-\mathrm{Cu}_{6} \mathrm{Sn}_{5}$ overall produced macroscopic tensile stress and its magnitude increased with the IMC growth. Similar to the previous section, it can also be found that pronounce tensile stress occurred at the grooves of two variants but slight compressive stress at the tips. The pattern of $\eta-\mathrm{Cu}_{6} \mathrm{Sn}_{5}$ displayed in Figure 5 seems to exhibit different alignments compared to that on $(001) \mathrm{Cu}$. The three variants align to three directions with an intersection angle of $120^{\circ}\left(\right.$ or $\left.60^{\circ}\right)$ between each other. According to the $\mathrm{L}_{2}$

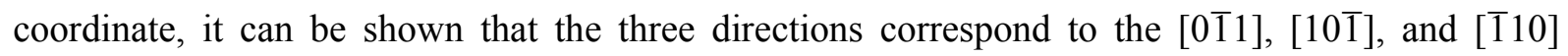
directions of $\mathrm{Cu}$, which is actually analogous to the growth on (001) $\mathrm{Cu}$. It can be further demonstrated that each direction also respectively reflects the principal direction of each heteroepitaxial strain tensor (Eq. (7)) with a minimal eigenvalue or atomic site mismatch. The growth

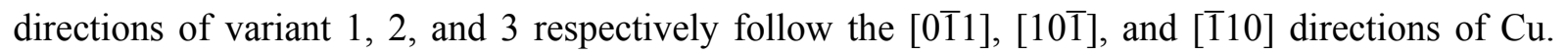
Because the best-matching direction can offer minimization of both interfacial energy and elastic 
strain energy, each variant can be energetically more favored to grow along the $<110>$ direction on both (001) and (111) $\mathrm{Cu}$.

\section{$4.33 \mathrm{D}$ simulation of $\eta-\mathrm{Cu}$ Sn 5 formation on (001) $\mathrm{Cu}$}

Figure 6(a) - (e) shows the time-evolution of $\eta-\mathrm{Cu}_{6} \mathrm{Sn}_{5}$ precipitated at the $\mathrm{Sn} /(001) \mathrm{Cu}$ interface. The distributions of $\mathrm{Cu}$ and liquid $\mathrm{Sn}$ phases are omitted in the figure for better demonstration. Because no $\eta-\mathrm{Cu}_{6} \mathrm{Sn}_{5}$ layer formed initially and worked as a diffusion barrier prior to interfacial reaction, the $\mathrm{Cu}$ atoms dissolved into liquid $\mathrm{Sn}$ rapidly and caused supersaturation of $\mathrm{Cu}$, especially for those close to the interface of $\mathrm{Cu}$ and liquid $\mathrm{Sn}$. The supersaturation provided a large chemical driving force for the precipitation of $\eta-\mathrm{Cu}_{6} \mathrm{Sn}_{5}$, as indicated in Figure 6(a). The magnitude of chemical driving force gradually decreased with the formation of $\eta-\mathrm{Cu}_{6} \mathrm{Sn}_{5}$ and the contribution of elastic energy became more significant, as pointed out by the stress field distribution in the $2 \mathrm{D}$ simulation. This situation was reflected by the specific pattern and alignment of $\eta-\mathrm{Cu}_{6} \mathrm{Sn}_{5}$ grains which gradually appeared during the latter stage of chemical reaction, as shown in Figure 6(b) - (e). The two directions correspond to the [110] and [110] directions of $\mathrm{Cu}$, according to the $\mathrm{L}_{1}$ coordinates chosen in the simulation on $(001) \mathrm{Cu}$. The result is consistent with the $2 \mathrm{D}$ simulation in Section 4.1. In addition to the preferred alignment, the coarsening phenomenon appeared at the same time, by which small grains were consumed gradually, as shown in Figure 6(b) - (e). The introduction of anisotropic interfacial energy (Section 3.4) results in the formation of faceted $\eta-\mathrm{Cu}_{6} \mathrm{Sn}_{5} / \mathrm{Sn}$ interface. Figure 6(f) is the experimental observation showing the top-view SEM (scanning electron microcopy) image of $\eta-\mathrm{Cu}_{6} \mathrm{Sn}_{5}$ on (001) single-crystal $\mathrm{Cu}$ [10]. The pattern and preferred growth directions predicted by the simulation exhibit excellent agreement with Figure 6(f).

Nevertheless, slight differences can be seen from the comparison of Figure 6(e) and 6(f). Although the simulated microstructure shows the faceted $\eta-\mathrm{Cu}_{6} \mathrm{Sn}_{5} / \mathrm{Sn}$ interface, the interface observed in experiment exhibited sharper edges and some of them grew more outward and normal 
from the surface. On the contrary, the simulated $\eta-\mathrm{Cu}_{6} \mathrm{Sn}_{5}$ was found to be more attached to the interface. This discrepancy may be caused by the simplified nucleation process and phenomenological treatment of anisotropic interfacial energy for accommodating the lack of experimental evaluations. Additionally, in the experimental work [10, 22], the samples had experienced a cooling process (to room temperature) after soldering reactions. The process produced even more precipitation of IMC on the interface due to the significantly lower solubility limit of Cu in Sn at room temperature. The current simulation work is not able to reflect the thermo-kinetic process of solidification, which is beyond the scope of this simulation study.

Figure 7 shows the pattern of $\eta-\mathrm{Cu}_{6} \mathrm{Sn}_{5}$ with each variant identified by different colors for visualizing each arrangement and distribution. It is readily seen again that the elongation of variant 1 aligns to the [110] direction of $\mathrm{Cu}$ lattice whereas that of variant 2 aligns the [110] direction. The simulation result with symmetry analysis of orientation variants is able to fully account for the speculation from experiments which showed $\eta-\mathrm{Cu}_{6} \mathrm{Sn}_{5}$ grains became elongated along the directions of small atomic spacing mismatch. The volume ratio of variant 1 and variant 2 shown in Figure 7 was calculated to be close to $1: 1$ since the two variants are both chemically and crystallographically equivalent. The zero-strain boundary condition prescribed at the $\mathrm{x}-\mathrm{y}$ plane also favors the 1:1 ratio of the two variants by canceling the off-diagonal or shear components of the heteroepitaxial strain tensors in Eq. (3). The cancelation makes the microstructure more compatible with the clamp boundary constraint.

\section{$4.43 \mathrm{D}$ simulation of $\eta-\mathrm{Cu} 6 \mathrm{Sn}_{5}$ formation on (111) $\mathrm{Cu}$}

The time-evolution of $\eta-\mathrm{Cu}_{6} \mathrm{Sn}_{5}$ formation on (111) $\mathrm{Cu}$ is shown in Figure 8(a) - (e). Figure 8(a) shows the early-stage nucleation without evident patterns or elongation, and Figure 8(b) shows the pattern formation and elongation of variants to three directions at the later stage, with several small grains formed in between those elongated variants. Some of the $\eta-\mathrm{Cu}_{6} \mathrm{Sn}_{5}$ grains were smaller than 
those found in the simulation on (001) $\mathrm{Cu}$ at the early stage. Similar to the previous section, coarsening of each $\eta-\mathrm{Cu}_{6} \mathrm{Sn}_{5}$ precipitates occurred and simultaneously the aligned growth of each variant was maintained. It can be demonstrated in Figure 8 that the preferred directions of the

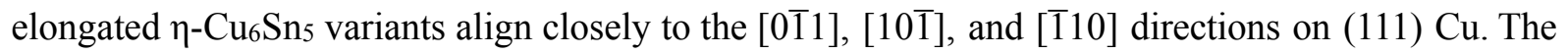
distribution of each variant is displayed in Figure 9, which shows that the aligned directions of variant

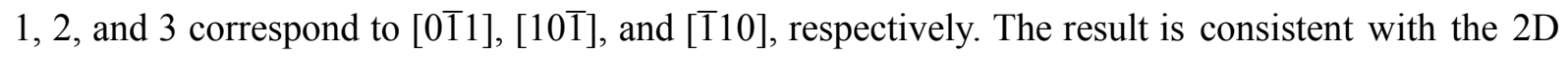
simulation (Section 4.2). Analogous to the previous section, each direction also respectively reflects the principal direction of each heteroepitaxial strain tensor (Eq. (7)) with a minimal eigenvalue or atomic site mismatch. Figure 8(f) shows the top-view SEM image of $\eta-\mathrm{Cu}_{6} \mathrm{Sn}_{5}$ on (111) single-crystal $\mathrm{Cu}$ [22]. The overall pattern and preferred growth directions in the simulation demonstrate consistency with that of the SEM result, though slight discrepancies as described in Section 4.3 were also found in the simulated growth of $\eta-\mathrm{Cu}_{6} \mathrm{Sn}_{5}$ on (111) $\mathrm{Cu}$.

From the comparison between Figure 7 and Figure 9 as well as in the experimental studies, it is shown that the interfacial reactions on (001) and (111) $\mathrm{Cu}$ demonstrate analogous pattern formations: All the elongated directions of $\eta-\mathrm{Cu}_{6} \mathrm{Sn}_{5}$ variants follow the dense $<110>$ rows on the $\mathrm{Cu}$ lattice planes, and accordingly the patterns can be determined basically by the directions those rows align with on the unidirectional surface of $\mathrm{Cu}$. It is worth noting that although the first part of the heteroepitaxial relationships on (001) $\mathrm{Cu}$ and $\eta-\mathrm{Cu}_{6} \mathrm{Sn}_{5}$ on (111) $\mathrm{Cu}$ are crystallographically different, the principal values of the total five heteroepitaxial strain tensors in Eq. (3) and Eq. (7) are exactly identical, which are $-0.51 \%$ and $-5.2 \%$, respectively. It is caused by the fact that the values of atomic site mismatch along the directions of $[110]_{\mathrm{Cu}} \|[\overline{1} 100]_{\eta}$ and $[11 \overline{2}]_{\mathrm{Cu}} \|[1 \overline{2} 10]_{\eta}$ are the same. In other words, the orientation variants of $\eta-\mathrm{Cu}_{6} \mathrm{Sn}_{5}$ on (001) and (111) $\mathrm{Cu}$ are mathematically equivalent, albeit based on nonequivalent crystallographic relationship, and thus the pattern formation on the two types of surface can operate in such a similar way. 


\section{5 $3 D$ simulation of $\eta-C u_{6} S_{5}$ formation without heteroepitaxial constraint}

Figure 10(a) - (e) shows the time evolution of $\eta-\mathrm{Cu}_{6} \mathrm{Sn}_{5}$ formation without elastic strain energy calculations in an attempt to reveal the effects of unidirectional $\mathrm{Cu}$ substrates and heteroepitaxial constraint shown in the previous sections. It is found that after initial nucleation, $\eta-\mathrm{Cu}_{6} \mathrm{Sn}_{5}$ grains are demonstrated to be of scalloped morphology without elongation along any specific direction. The scalloped shape of $\eta-\mathrm{Cu}_{6} \mathrm{Sn}_{5}$ grains with deep grooves at $\eta-\mathrm{Cu}_{6} \mathrm{Sn}_{5}$ grain boundaries was attributed to the smaller interfacial energy of $\eta-\mathrm{Cu}_{6} \mathrm{Sn}_{5} / \mathrm{Sn}$ compared to that of $\eta-\mathrm{Cu}_{6} \mathrm{Sn}_{5}$ grain boundaries [1]. From the energetic perspective, the deep grooves of $\eta-\mathrm{Cu}_{6} \mathrm{Sn}_{5}$ grains increased the area of the $\eta-\mathrm{Cu}_{6} \mathrm{Sn}_{5} \mathrm{Sn}$ interface with lower interfacial energy. The evolution of microstructures was solely controlled by the interfacial-energy-driven ripening or coarsening process. The result is similar to the 3D phase-field simulation work of IMC layer growth by Kim et al. [73]. Figure 10(f) shows the experimental observation of $\eta-\mathrm{Cu}_{6} \mathrm{Sn}_{5}$ grain morphology and it can be found that the simulation is consistent with the previous phase-field simulation [73] and experiment [22].

\section{DISCUSSION}

\subsection{Analysis of habit planes}

Our simulation results have successfully reproduced the pattern formation of $\eta-\mathrm{Cu}_{6} \mathrm{Sn}_{5}$ precipitates on (001) and (111) planes of $\mathrm{Cu}$ in both two and three dimensions. In this section, we analyze some of the detailed morphological characteristics of the patterns. We will start with calculating quantitatively the habit plane normal of each variant using the microelasticity theory.

The habit plane normal can be determined by searching for a unit vector $\mathbf{n}=\mathbf{n}_{0}$ in reciprocal space, which corresponds to the normal of the precipitate habit plane in real space, such that the function $B_{p q}\left(\mathbf{n}_{0}\right)$ reaches the minimum value. $B_{p q}(\mathbf{n})$, defined by Eq. (12), characterizes the coherency strain energy density (in reciprocal space) and is expressed as [29] 


$$
E^{\text {elas }}=\frac{1}{2} \sum_{p, q} \int \frac{d^{3} g}{(2 \pi)^{3}} B_{p q}(\mathbf{n}) \tilde{\phi}_{p}(\mathbf{g}) \tilde{\phi}_{q}^{*}(\mathbf{g})
$$

and

$$
B_{p q}(\mathbf{n})=\left\{\begin{array}{cc}
C_{i j k l} \varepsilon_{i j}^{00}(p) \varepsilon_{k l}^{00}(q) & \mathbf{n}=0 \\
C_{i j k l} \varepsilon_{i j}^{00}(p) \varepsilon_{k l}^{00}(q)-n_{i} \tilde{\sigma}_{i j}^{00}(p) \Omega_{j k}^{0} \tilde{\sigma}_{k l}^{00}(q) n_{l} & \mathbf{n} \neq 0
\end{array}\right.
$$

for the clamp boundary condition, where $p$ and $q$ specify the orientation variants, $\tilde{\phi}_{q}^{*}$ is the complex conjugate of $\tilde{\phi}_{q}$, and $\tilde{\sigma}_{i j}^{00}=C_{i j k l} \tilde{\varepsilon}_{k l}^{00}$.

Figure 11(a) and 11(b) show the polar plots of $B_{p p}(\mathbf{n})$ projected on the (001) and (111) planes of $\mathrm{Cu}$ based on $\mathrm{L}_{1}$ and $\mathrm{L}_{2}$ coordinates, respectively. It is seen that for the two variants on $(001) \mathrm{Cu}, B_{11}$ and $B_{22}$ reach minimum when the habit plane normal $\mathbf{n}_{0}$ of each variant corresponds to the [1 $\left.\overline{1} 0\right]$ and [110] directions, respectively, as indicated by the arrows. Similarly, $B_{11}, B_{22}$, and $B_{33}$ of the three

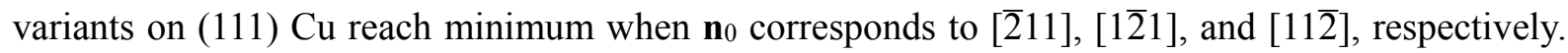
Thus for all the $\eta-\mathrm{Cu}_{6} \mathrm{Sn}_{5}$ variants on the (001) and (111) planes of $\mathrm{Cu}$, their growth along the $<110>_{\mathrm{Cu}}$ $\|[0001]_{\eta}$ directions will yield habit planes of each variant as predicted by the analysis of $B_{p p}(\mathbf{n})$. If variant 1 on (111) $\mathrm{Cu}$ is considered, for example, its growth along the [011] direction results in a ( $\overline{2} 11)$ habit plane, which is exactly the habit plane predicted by the minimization of $B_{11}$. It can be shown in Section 4.1 and 4.2 that the habit planes on both the (001) and (111) planes are consistent with those predicted by the minimization of $B_{p p}(\mathbf{n})$. This analysis indicates that the observed patterns in the simulations are dominated by the minimization of the coherency elastic strain energy.

\subsection{Analysis of kinematic compatibility}

In addition to the habit planes and directions of growth, it is also important to analyze the lattice continuity across the interface between two $\eta-\mathrm{Cu}_{6} \mathrm{Sn}_{5}$ variants on a unidirectional $\mathrm{Cu}$ substrate. The condition was described mathematically by the condition of kinematic or rank-one compatibility [74]. 


$$
\mathbf{Q} \mathbf{F}_{i}-\mathbf{F}_{j}=\mathbf{a} \otimes \hat{\mathbf{n}}
$$

where $\mathbf{Q}$ is a rigid body rotation matrix of variant $i$ with respect to variant $j, \mathbf{a}$ is a shear vector, $\hat{\mathbf{n}}$ is the normal unit vector of the interface between variants $i$ and $j$, and $\otimes$ is a dyadic product. Since the heteroepitaxial descriptions in Section 2.2 and 2.3 are formulated in $2 \mathrm{D}$ at the $\eta-\mathrm{Cu}_{6} \mathrm{Sn}_{5} / \mathrm{Cu}$ interface, the compatibility condition is examined in 2D as well. The solution of Eq. (20) has been given by Ball and James [74].

For the growth of two $\eta-\mathrm{Cu}_{6} \mathrm{Sn}_{5}$ variants on $(001) \mathrm{Cu}$, if $i$ and $j$ correspond respectively to variant 1 and variant 2, there exists a set of two solutions, $\hat{\mathbf{n}}=[0,1]_{\mathrm{L} 1}$ or $\hat{\mathbf{n}}=[1,0]_{\mathrm{L} 1}$ with respect to the $\mathrm{L}_{1}$ coordinate. The normal direction of the variant 1/variant 2 interface has to be either along the [001] or [010] direction to keep the $\eta-\mathrm{Cu}_{6} \mathrm{Sn}_{5}$ lattices continuous across the interface. It is noted that the same set of solution can also be given by the geometrically linear theory based on strain compatibility [75]:

$$
\boldsymbol{\varepsilon}^{00}(1)-\boldsymbol{\varepsilon}^{00}(2)=\frac{1}{2}(\mathbf{a} \otimes \hat{\mathbf{n}}+\hat{\mathbf{n}} \otimes \mathbf{a})
$$

or precisely,

$$
\begin{aligned}
\boldsymbol{\varepsilon}^{00}(1)-\boldsymbol{\varepsilon}^{00}(2) & =\frac{1}{2}\{[0.094,0] \otimes[0,1]+[0,1] \otimes[0.094,0]\} \\
& =\frac{1}{2}\{[0,0.094] \otimes[1,0]+[1,0] \otimes[0,0.094]\}
\end{aligned}
$$

The solution can be validated by the $2 \mathrm{D}$ simulations of growth on $(001) \mathrm{Cu}$, as shown by the dash lines in Figure 4. The normal direction of the variant 1/variant 2 interface gradually approaches to the $[0,1] \mathrm{L} 1$ or $[1,0]_{\mathrm{L} 1}$ direction. This characteristic is in excellent agreement with the prediction of the kinematic compatibility.

It is also straightforward to solve the compatibility condition of the three $\eta-\mathrm{Cu}_{6} \mathrm{Sn} 5$ variants on (111) $\mathrm{Cu}$ with respect to the $\mathrm{L}_{2}$ coordinate. For the interface of variant 1 / variant 2 , variant 2 / variant 3 , and variant $1 /$ variant 3 , the solutions give $\hat{\mathbf{n}}=[0.866,-0.500]_{\mathrm{L} 2}$ or $\hat{\mathbf{n}}=[0.500,0.866]_{\mathrm{L} 2}, \hat{\mathbf{n}}=[0$, $1]_{\mathrm{L} 2}$ or $\hat{\mathbf{n}}=[1,0]_{\mathrm{L} 2}$, and $\hat{\mathbf{n}}=[0.866,0.500]_{\mathrm{L} 2}$ or $\hat{\mathbf{n}}=[-0.500,0.866]_{\mathrm{L} 2}$, respectively. The common feature of the solutions on (001) and (111) $\mathrm{Cu}$ is that the bisector direction of any two preferred 
growth directions $\left(<110>_{\mathrm{Cu}}\right)$ on $(001)$ or $(111) \mathrm{Cu}$ is able to align on one of the interfaces predicted by kinematic compatibility. If considering the interface of variant $1 /$ variant 2 , the solution of compatibility reflects the interface with a normal vector $\hat{\mathbf{n}}=[\overline{110}]$ or $\hat{\mathbf{n}}=[\overline{11} 2]$ of $\mathrm{Cu}$. The two bisector directions of variant 1 and variant 2 can be found to align on these two interfaces. The coherent interfacial characteristic has not been identified by experiments, but it can be found in good agreement with 2D simulations, as demonstrated by the variant boundaries in Figure 4 and Figure 5.

\subsection{Comparison of OR predictions and recent experiments}

As described in Section 2.1, the edge-to-edge matching model predicts two types of OR, [0001] $\left\|[110]_{\mathrm{Cu}},(1 \overline{2} 10)_{\eta}\right\|(1 \overline{1} 1)_{\mathrm{Cu}}\left(\right.$ Type I), and $[0001]_{\eta}\left\|[110]_{\mathrm{Cu}},(0 \overline{1} 10)_{\eta}\right\|(100)_{\mathrm{Cu}}$ (Type II), both of which share the same directional relation. The crystallographic relationship between $\eta-\mathrm{Cu}_{6} \mathrm{Sn}_{5}$ and $\mathrm{Cu}$ was studied recently by a combined analysis of EBSD and TEM [56], though not explicitly addressed and pointed out by the authors, both Type I and II ORs can actually been observed by the SAED (selected area electron diffraction) images. For the $\eta-\mathrm{Cu}_{6} \mathrm{Sn}_{5}$ growth on (001) $\mathrm{Cu}$, the SAED pattern from the zone axis $[0001]_{\eta} \|[110]_{\mathrm{Cu}}$ showed that the $(1 \overline{2} 10)_{\eta}$ plane was in near parallel to $(1 \overline{1}$ 1) $\mathrm{Cu}$ with a rotation angle of $5.26^{\circ}$ about the matching direction $[0001]_{\eta} \|[110]_{\mathrm{Cu}}$. Similarly for the $\eta-\mathrm{Cu}_{6} \mathrm{Sn}_{5}$ growth on (111) $\mathrm{Cu}$, the SAED pattern from the same zone axis showed that the $(0 \overline{1} 10)_{\eta}$ plane was in near parallel to $(100)_{\mathrm{Cu}}$ with a rotation of $5.26^{\circ}$. It is noted that from the viewpoint of the edge-to-edge matching model, the small rotation angle is required to let the interface of two phases meeting edge-to-edge and possess a maximum matching of atomic rows and a lower interfacial energy. The SAED images indicate that the $\eta-\mathrm{Cu}_{6} \mathrm{Sn}_{5}$ growth on (001) $\mathrm{Cu}$ follows Type I OR, whereas that on (111) Cu follows Type II OR. The SAED result from another previous study also showed that the $\eta-(\mathrm{Cu}, \mathrm{Ni})_{6} \mathrm{Sn}_{5}$ growth on $(001) \mathrm{Ni}$ followed Type I OR, in accord with the growth on $(001) \mathrm{Cu}$. It is possible that the OR on a specific surface of the substrate is chosen in a way to release the in-plane heteroepitaxial constraint or have a lowest possible strain of the initial layer [59]. Nevertheless, 
fundamental rationales behind the differently chosen ORs between the IMCs and $\mathrm{Cu}$ substrate of different orientations still remain ambiguous and require further experimental and theoretical investigations. The work should be essential for understanding and predictions of IMC pattern formation.

\subsection{Potential applications and limitations of the model}

One of the most important advantages of the present model is its ability to be extended to simulate the growth of materials on other substrates of any oriented direction as long as the heteroepitaxial relationship or correspondence is identified. It should be pointed out that the number of variants and their growth patterns are not pre-assumed in this model. Instead, the variant is determined by following the principle that multiple variants will be generated when the material is grown on a unidirectional substrate of higher symmetry [36], and the heteroepitaxial strain of each variant is characterized by the lattice correspondence suggested by the experimentally observed OR. Finally, the overall precipitate patterns are controlled by the heteroepitaxial constraint on the unidirectional substrates as compared to the IMC formed on polycrystalline $\mathrm{Cu}$ without the elastic strain energy considered. The successful application and agreement with experimental observations on both (001) and (111) Cu clearly demonstrate its applicability. It should be a straightforward exercise to apply the model to other solder systems such as Ni-Sn and Ag-Sn. Experiments have shown that IMC growth on unidirectional Ag or Ni produced distinctive morphological features as well $[24,76]$, including the growth of $\mathrm{Ni}_{3} \mathrm{Sn}_{4}$ (monoclinic, $C 2 / m$ [77]) on $\mathrm{Ni}$ and $\mathrm{Ag}_{3} \mathrm{Sn}$ (orthorhombic, Pmmn [78]) on Ag. Because of the different crystal structures of the IMCs, the orientation variants on a unidirectional substrate, their ORs and heteroepitaxial correspondence are required to be re-determined and re-evaluated by following Section 2. The methodology developed in this study also provides a robust approach to model pattern formations on heteroepitaxial or epitaxial thin films and offer insights into the establishment of structure-morphology relationships. 
Nevertheless, the current model has some limitations. As mentioned in Section 3.6, homogeneous and isotropic elastic modulus of $\eta-\mathrm{Cu}_{6} \mathrm{Sn}_{5}$ is assumed for simplicity. The nucleation process was simulated by the Langevin-noise terms introduced at the liquid and substrate interface, which is qualitative in nature but may be sufficient for the study of the strain-induced pattern formation. Additionally, only the interfacial energy of the $\eta-\mathrm{Cu}_{6} \mathrm{Sn}_{5} / \mathrm{Sn}$ interface is treated to be anisotropic, whereas those of the other interfaces are assumed isotropic. Since the present work is aimed to establish a general model for heteroepitaxial IMC growth and focuses on the effect of heteroepitaxial strain on IMC pattern formation, we leave the consideration of anisotropic and inhomogeneous elasticity for future development of more accurate models. Despite these limitations, the present simulation results are in excellent agreement with the pattern formations observed in experiments, which are essentially strain-induced. The application of this approach is not limited to interfacial reactions in solder interconnects, but can be extended to any heteroepitaxial growth of materials in an analogous manner.

\section{CONCLUSION}

Pattern formation and heteroepitaxial growth of $\eta-\mathrm{Cu}_{6} \mathrm{Sn}_{5}$ during chemical reactions between $\mathrm{Sn}$ and unidirectional (001) and (111) $\mathrm{Cu}$ are investigated by using a combination of crystallographic analysis, microelasticity analysis and phase-field simulations in both $2 \mathrm{D}$ and $3 \mathrm{D}$. The symmetry-related orientation variants determined by the crystallographic analysis are characterized by a set of non-conserved order parameters in the phase-field model. According to the analysis, two and three $\eta-\mathrm{Cu}_{6} \mathrm{Sn}_{5}$ variants can be generated on (001) and (111)-oriented $\mathrm{Cu}$ single crystal substrates,

respectively. For each variant, the stress-free heteroepitaxial strain is calculated quantitatively based on the identified heteroepitaxial relationship and symmetry operations.

The $\eta-\mathrm{Cu}_{6} \mathrm{Sn}_{5}$ domain patterns on both (001) and (111) $\mathrm{Cu}$ obtained from the phase-field simulations show excellent agreements with the experimental observations. The results on (001) $\mathrm{Cu}$ 
show that the $\eta-\mathrm{Cu}_{6} \mathrm{Sn}_{5}$ grains grow along two perpendicular directions, while the results on (111) $\mathrm{Cu}$ show that the $\eta-\mathrm{Cu}_{6} \mathrm{Sn}_{5}$ grains growth along three directions with an intersection angles of $120^{\circ}$ or $60^{\circ}$. It can be seen that each $\eta-\mathrm{Cu}_{6} \mathrm{Sn}_{5}$ variant, either on (001) or (111) $\mathrm{Cu}$, grows along the principal direction of heteroepitaxial strain with the minimum eigenvalue, which in all the cases corresponds to the $<110>_{\mathrm{Cu}}$ direction. In contrast, $\eta-\mathrm{Cu}_{6} \mathrm{Sn}_{5}$ grains grown without the heteroepitaxial constraints (e.g., grown on a polycrystalline substrate) do not exhibit any alignment. Additionally, the consideration of anisotropic interfacial energy correctly predicts faceted $\eta-\mathrm{Cu}_{6} \mathrm{Sn}_{5} / \mathrm{Sn}$ interface as observed in experiments.

The alignment along $\left\langle 110>_{\mathrm{Cu}}\right.$ found in the phase-field simulations is further elucidated by the microelasticity analysis, which is shown to minimize $B_{p p}(\mathbf{n})$, the characteristic coherency elastic energy density. The interface normal of any two $\eta-\mathrm{Cu}_{6} \mathrm{Sn}_{5}$ variants observed in the simulations as well as in the experiments is found to obey the kinematic compatibility condition. The stress field calculations based on 2D simulations show that strong tensile stress can be generated at the grooves of the $\eta-\mathrm{Cu}_{6} \mathrm{Sn}_{5}$ variant boundaries.

Two types of orientation relationships (ORs) between $\eta-\mathrm{Cu}_{6} \mathrm{Sn}_{5}$ and $\mathrm{Cu}$ substrates are predicted by the edge-to-edge matching model, which are actually appeared in the SAED pattern from a recent study. The ORs for $\eta-\mathrm{Cu}_{6} \mathrm{Sn}_{5}$ growth on (001) and (111) $\mathrm{Cu}$ are identified to be $[0001]_{\eta} \|[110]_{\mathrm{Cu}},(1 \overline{2}$ 10) $\|(1 \overline{1} 1)_{\mathrm{Cu}}$ and $[0001]_{\eta}\left\|[110]_{\mathrm{Cu}},(0 \overline{1} 10)_{\eta}\right\|(100)_{\mathrm{Cu}}$, respectively.

ACKNOWLEDGEMENTS: J.H.K. and C.R.K. acknowledge the financial support from National Science Council of Taiwan through Grant NSC-101-2917-I-002-016. Part of this work was done while the author (J.H.K.) was at Ohio State University. Y.G. and Y.W. acknowledge the financial support from National Science Foundation of the United States through Grant No. DMR-1410322. We thank Prof. K. N. Tu (Department of Materials Science and Engineering, UCLA) and Prof. Z. F. Zhang (Shenyang National Laboratory for Materials Science) for permission to use published 
materials. J.H.K. would like to thank Prof. Y. C. Shu (Institute of Applied Mechanics, National Taiwan University), Dr. R. Shi (Department of Materials Science and Engineering, The Ohio State University) for valuable discussions, and Prof. N. Moelans (Department of Materials Engineering, Katholieke Universiteit Leuven) for sharing thermodynamic parameters. 


\section{REFERENCES}

[1] K. N. Tu, Solder Joint Technology: Materials, Properties, and Reliability. (Springer, 2007)

[2] K. N. Tu, R. D. Thompson, Kinetics of interfacial reaction in bimetallic $\mathrm{Cu}-\mathrm{Sn}$ thin films, Acta Metallurgica 30 (1982) 947-952.

[3] U. Gösele, K. N. Tu, Growth kinetics of planar binary diffusion couples: "Thin-film case" versus 'bulk cases", Journal of Applied Physics 53 (1982) 3252-3260.

[4] S. Bader, W. Gust, and H. Hieber, Rapid formation of intermetallic compounds interdiffusion in the Cu-Sn and Ni-Sn systems, Acta Metallurgica et Materialia 43 (1995) 329-337.

[5] T. Laurila, V. Vuorinen, and J. K. Kivilahti, Interfacial reactions between lead-free solders and common base materials, Materials Science and Engineering: R: Reports 49 (2005) 1-60.

[6] C. E. Ho, S. C. Yang, and C. R. Kao, Interfacial reaction issues for lead-free electronic solders, Journal of Materials Science-Materials in Electronics 18 (2007) 155-174.

[7] Q. Y. Yin, F. Gao, Z. Y. Gu, E. A. Stach, and G. W. Zhou, In situ visualization of metallurgical reactions in nanoscale $\mathrm{Cu} / \mathrm{Sn}$ diffusion couples, Nanoscale 7 (2015) 4984-4994.

[8] J. O. Suh, K. N. Tu, G. V. Lutsenko, and A. M. Gusak, Size distribution and morphology of $\mathrm{Cu}_{6} \mathrm{Sn}_{5}$ scallops in wetting reaction between molten solder and copper, Acta Materialia 56 (2008) 1075-1083.

[9] J. O. Suh, K. N. Tu, and N. Tamura, Preferred orientation relationship between $\mathrm{Cu}_{6} \mathrm{Sn}_{5}$ scallop-type grains and $\mathrm{Cu}$ substrate in reactions between molten $\mathrm{Sn}$-based solders and $\mathrm{Cu}$, Journal of Applied Physics 102 (2007) 063511.

[10] J. O. Suh, K. N. Tu, and N. Tamura, Dramatic morphological change of scallop-type $\mathrm{Cu}_{6} \mathrm{Sn}_{5}$ formed on (001) single crystal copper in reaction between molten $\mathrm{SnPb}$ solder and $\mathrm{Cu}$, Applied Physics Letters 91 (2007) 051907.

[11] K. H. Prakash, T. Sritharan, Interface reaction between copper and molten tin-lead solders, Acta Materialia 49 (2001) 2481-2489.

[12] H. K. Kim, K. N. Tu, Kinetic analysis of the soldering reaction between eutectic $\mathrm{SnPb}$ alloy 
and $\mathrm{Cu}$ accompanied by ripening, Physical Review B 53 (1996) 16027-16034.

[13] K. N. Tu, Recent advances on electromigration in very-large-scale-integration of interconnects, Journal of Applied Physics 94 (2003) 5451-5473.

[14] J. H. Ke, T. L. Yang, Y. S. Lai, and C. R. Kao, Analysis and experimental verification of the competing degradation mechanisms for solder joints under electron current stressing, Acta Materialia 59 (2011) 2462-2468.

[15] J. H. Ke, H. Y. Chuang, W. L. Shih, and C. R. Kao, Mechanism for serrated cathode dissolution in $\mathrm{Cu} / \mathrm{Sn} / \mathrm{Cu}$ interconnect under electron current stressing, Acta Materialia 60 (2012) 2082-2090.

[16] F. Ren, J.-W. Nah, K. N. Tu, B. Xiong, L. Xu, and J. H. L. Pang, Electromigration induced ductile-to-brittle transition in lead-free solder joints, Applied Physics Letters 89 (2006) 141914.

[17] G. Ghosh, Elastic properties, hardness, and indentation fracture toughness of intermetallics relevant to electronic packaging, Journal of Materials Research 19 (2004) 1439-1454.

[18] L. Jiang, N. Chawla, Mechanical properties of $\mathrm{Cu}_{6} \mathrm{Sn}_{5}$ intermetallic by micropillar compression testing, Scripta Materialia 63 (2010) 480-483.

[19] L. Zhang, K. N. Tu, Structure and properties of lead-free solders bearing micro and nano particles, Materials Science and Engineering: R: Reports 82 (2014) 1-32.

[20] H. Y. Hsiao, C. M. Liu, H. W. Lin, T. C. Liu, C. L. Lu, Y. S. Huang, C. Chen, and K. N. Tu, Unidirectional Growth of Microbumps on (111)-Oriented and Nanotwinned Copper, Science 336 (2012) 1007-1010.

[21] T. C. Liu, C. M. Liu, H. Y. Hsiao, J. L. Lu, Y. S. Huang, and C. Chen, Fabrication and Characterization of (111)-Oriented and Nanotwinned $\mathrm{Cu}$ by Dc Electrodeposition, Crystal Growth \& Design 12 (2012) 5012-5016.

[22] H. F. Zou, H. J. Yang, and Z. F. Zhang, Morphologies, orientation relationships and evolution of $\mathrm{Cu}_{6} \mathrm{Sn}_{5}$ grains formed between molten $\mathrm{Sn}$ and $\mathrm{Cu}$ single crystals, Acta Materialia 56 (2008) 
$2649-2662$.

[23] W. M. Chen, T. L. Yang, C. K. Chung, and C. R. Kao, The orientation relationship between Ni and $\mathrm{Cu}_{6} \mathrm{Sn}_{5}$ formed during the soldering reaction, Scripta Materialia 65 (2011) 331-334.

[24] H. F. Zou, H. J. Yang, J. Tan, and Z. F. Zhang, Preferential growth and orientation relationship of $\mathrm{Ag}_{3} \mathrm{Sn}$ grains formed between molten $\mathrm{Sn}$ and (001) Ag single crystal, Journal of Materials Research 24 (2009) 2141-2144.

[25] F. F. Tian, Z. Q. Liu, Growth mechanism of duplex structural $\mathrm{Cu}_{2}(\mathrm{In}, \mathrm{Sn})$ compound on single crystalline Cu substrate, Journal of Alloys and Compounds 588 (2014) 662-667.

[26] M. X. Zhang, P. M. Kelly, Crystallographic features of phase transformations in solids, Progress in Materials Science 54 (2009) 1101-1170.

[27] H. Gao, Some general properties of stress-driven surface evolution in a heteroepitaxial thin film structure, Journal of the Mechanics and Physics of Solids 42 (1994) 741-772.

[28] H. Gao, W. D. Nix, Surface roughening of heteroepitaxial thin films, Annual Review of Materials Science 29 (1999) 173-209.

[29] A. Khachaturian, Theory of structural transformations in solids. (John Wiley and Sons, New York, 1983)

[30] N. Moelans, B. Blanpain, and P. Wollants, An introduction to phase-field modeling of microstructure evolution, Calphad-Computer Coupling of Phase Diagrams and Thermochemistry 32 (2008) 268-294.

[31] I. Steinbach, B. Bottger, J. Eiken, N. Warnken, and S. G. Fries, CALPHAD and phase-field modeling: A successful liaison, Journal of Phase Equilibria and Diffusion 28 (2007) 101-106.

[32] J. Z. Zhu, Z. K. Liu, V. Vaithyanathan, and L. Q. Chen, Linking phase-field model to CALPHAD: application to precipitate shape evolution in Ni-base alloys, Scripta Materialia 46 (2002) 401-406.

[33] M. S. Park, R. Arroyave, Early stages of intermetallic compound formation and growth during lead-free soldering, Acta Materialia 58 (2010) 4900-4910. 
[34] M. S. Park, R. Arróyave, Concurrent nucleation, formation and growth of two intermetallic compounds $\left(\mathrm{Cu}_{6} \mathrm{Sn}_{5}\right.$ and $\left.\mathrm{Cu}_{3} \mathrm{Sn}\right)$ during the early stages of lead-free soldering, Acta Materialia 60 (2012) 923-934.

[35] M. S. Park, S. L. Gibbons, and R. Arróyave, Phase-field simulations of intermetallic compound evolution in $\mathrm{Cu} / \mathrm{Sn}$ solder joints under electromigration, Acta Materialia 61 (2013) 7142-7154.

[36] C. P. Flynn, J. A. Eades, Structural variants in heteroepitaxial growth, Thin Solid Films 389 (2001) 116-137.

[37] K. K. Wang, D. S. Gan, and K. C. Hsieh, Orientation relationships, interfaces, and microstructure of $\eta-\mathrm{Cu}_{6} \mathrm{Sn}_{5}$ formed in the early-stage reaction between $\mathrm{Cu}$ and molten $\mathrm{Sn}$, Thin Solid Films 519 (2010) 1380-1386.

[38] Y. Wang, L.-Q. Chen, and A. G. Khachaturyan, Shape evolution of a precipitate during strain-induced coarsening: A computer simulation, Scripta Metallurgica et Materialia 25 (1991) 1387-1392.

[39] L.-Q. Chen, Y. Wang, and A. G. Khachaturyan, Transformation-induced elastic strain effect on the precipitation kinetics of ordered intermetallics, Philosophical Magazine Letters 64 (1991) 241-251.

[40] Y. Wang, A. G. Khachaturyan, Three-dimensional field model and computer modeling of martensitic transformations, Acta Materialia 45 (1997) 759-773.

[41] C. Shen, Y. Wang, in Handbook of materials modeling. (Springer, 2005), pp. 2117-2142.

[42] A. Boyne, M. D. Rauscher, S. A. Dregia, and Y. Wang, Surface island formation with localized stresses, Scripta Materialia 64 (2011) 705-708.

[43] A. Boyne, S. A. Dregia, and Y. Wang, Concurrent spinodal decomposition and surface roughening in thin solid films, Applied Physics Letters 99 (2011) 063111.

[44] S. M. Wise, J. S. Lowengrub, J. S. Kim, and W. C. Johnson, Efficient phase-field simulation of quantum dot formation in a strained heteroepitaxial film, Superlattices and Microstructures 
36 (2004) 293-304.

[45] S. M. Wise, W. C. Johnson, Numerical simulations of pattern-directed phase decomposition in a stressed, binary thin film, Journal of Applied Physics 94 (2003) 889-898.

[46] W. Lu, Z. Suo, Dynamics of nanoscale pattern formation of an epitaxial monolayer, Journal of the Mechanics and Physics of Solids 49 (2001) 1937-1950.

[47] C. Folkman, S. Baek, H. Jang, C. Eom, C. Nelson, X. Pan, Y. Li, L. Chen, A. Kumar, and V. Gopalan, Stripe domain structure in epitaxial (001) $\mathrm{BiFeO}_{3}$ thin films on orthorhombic $\mathrm{TbScO}_{3}$ substrate, Applied Physics Letters 94 (2009) 251911.

[48] G. Sheng, J. X. Zhang, Y. L. Li, S. Choudhury, Q. X. Jia, Z. K. Liu, and L. Q. Chen, Misfit strain-misfit strain diagram of epitaxial $\mathrm{BaTiO}_{3}$ thin films: Thermodynamic calculations and phase-field simulations, Applied Physics Letters 93 (2008) 232904.

[49] J. Zhang, Y. Li, D. Schlom, L. Chen, F. Zavaliche, R. Ramesh, and Q. Jia, Phase-field model for epitaxial ferroelectric and magnetic nanocomposite thin films, Applied Physics Letters 90 (2007) 052909.

[50] Y. U. Wang, Y. M. Jin, A. M. Cuitiño, and A. G. Khachaturyan, Nanoscale phase field microelasticity theory of dislocations: model and 3D simulations, Acta Materialia 49 (2001) 1847-1857.

[51] Y. Wang, J. Li, Phase field modeling of defects and deformation, Acta Materialia 58 (2010) $1212-1235$

[52] J. H. Ke, A. Boyne, Y. Wang, and C. R. Kao, Phase field microelasticity model of dislocation climb: Methodology and applications, Acta Materialia 79 (2014) 396-410.

[53] D. A. Cogswell, M. Z. Bazant, Coherency Strain and the Kinetics of Phase Separation in $\mathrm{LiFePO}_{4}$ Nanoparticles, Acs Nano 6 (2012) 2215-2225.

[54] T. Ichitsubo, K. Tokuda, S. Yagi, M. Kawamori, T. Kawaguchi, T. Doi, M. Oishi, and E. Matsubara, Elastically constrained phase-separation dynamics competing with the charge process in the $\mathrm{LiFePO}_{4} / \mathrm{FePO}_{4}$ system, Journal of Materials Chemistry A 1 (2013) 2567-2577. 
[55] N. Saunders, A. P. Miodownik, The Cu-Sn (Copper-Tin) system, Bulletin of Alloy Phase Diagrams 11 (1990) 278-287.

[56] Z. H. Zhang, M. Y. Li, Z. Q. Liu, and S. H. Yang, Growth characteristics and formation mechanisms of $\mathrm{Cu}_{6} \mathrm{Sn}_{5}$ phase at the liquid-Sn0.7Cu/(111) Cu and liquid-Sn0.7Cu/(001)Cu joint interfaces, Acta Materialia 104 (2016) 1-8.

[57] J. W. Cahn, G. Kalonji, in Proc. Intl. Conf. on Solid-Solid Phase Transformations.

[58] Vantende.G, Amelinck.S, Group-Theoretical Considerations Concerning Domain Formation in Ordered Alloys, Acta Crystallographica Section A A 30 (1974) 431-440.

[59] J. Griesche, R. Enderlein, and D. Schikora, Orientation and strain in heteroepitaxial growth, physica status solidi (a) 109 (1988) 11-38.

[60] A. C. Hillier, M. D. Ward, Epitaxial interactions between molecular overlayers and ordered substrates, Physical Review B 54 (1996) 14037-14051.

[61] D. E. Hooks, T. Fritz, and M. D. Ward, Epitaxy and Molecular Organization on Solid Substrates, Advanced Materials 13 (2001) 227-241.

[62] I. Steinbach, F. Pezzolla, B. Nestler, M. Seesselberg, R. Prieler, G. J. Schmitz, and J. L. L. Rezende, A phase field concept for multiphase systems, Physica D 94 (1996) 135-147.

[63] I. Steinbach, F. Pezzolla, A generalized field method for multiphase transformations using interface fields, Physica D 134 (1999) 385-393.

[64] M. S. Park, S. L. Gibbons, and R. Arróyave, Phase-field simulations of intermetallic compound growth in $\mathrm{Cu} / \mathrm{Sn} / \mathrm{Cu}$ sandwich structure under transient liquid phase bonding conditions, Acta Materialia 60 (2012) 6278-6287.

[65] K. W. Moon, W. J. Boettinger, U. R. Kattner, F. S. Biancaniello, and C. A. Handwerker, Experimental and thermodynamic assessment of $\mathrm{Sn}-\mathrm{Ag}-\mathrm{Cu}$ solder alloys, Journal of Electronic Materials 29 (2000) 1122-1136.

[66] N. Moelans, A. Kroupa, Ed. (COST, 2012).

[67] S. G. Kim, W. T. Kim, and T. Suzuki, Phase-field model for binary alloys, Physical Review E 
60 (1999) 7186-7197.

[68] F. Wendler, C. Mennerich, and B. Nestler, A phase-field model for polycrystalline thin film growth, Journal of Crystal Growth 327 (2011) 189-201.

[69] Y. Wang, H.-Y. Wang, L.-Q. Chen, and A. G. Khachaturyan, Microstructural Development of Coherent Tetragonal Precipitates in Magnesium-Partially-Stabilized Zirconia: A Computer Simulation, Journal of the American Ceramic Society 78 (1995) 657-661.

[70] J. W. Cahn, J. E. Hilliard, Free Energy of a Nonuniform System. I. Interfacial Free Energy, The Journal of Chemical Physics 28 (1958) 258-267.

[71] V. Kumar, Z. Z. Fang, J. Liang, and N. Dariavach, Microstructural analysis of lead-free solder alloys, Metallurgical and Materials Transactions A 37 (2006) 2505-2514.

[72] Y. Tian, R. Zhang, C. Hang, L. Niu, and C. Wang, Relationship between morphologies and orientations of Cu6Sn5 grains in $\mathrm{Sn} 3.0 \mathrm{Ag} 0.5 \mathrm{Cu}$ solder joints on different $\mathrm{Cu}$ pads, Materials Characterization 88 (2014) 58-68.

[73] H.-S. Kim, H.-J. Lee, Y.-S. Yu, and Y.-S. Won, Three-dimensional simulation of intermetallic compound layer growth in a binary alloy system, Acta Materialia 57 (2009) 1254-1262.

[74] J. M. Ball, R. D. James, Fine phase mixtures as minimizers of energy, Archive for Rational Mechanics and Analysis 100 (1987) 13-52.

[75] K. Bhattacharya, Comparison of the geometrically nonlinear and linear theories of martensitic transformation, Continuum Mechanics and Thermodynamics 5 (1993) 205-242.

[76] J. Suh, K. Tu, A. T. Wu, and N. Tamura, Preferred orientation relationships with large misfit interfaces between $\mathrm{Ni}_{3} \mathrm{Sn}_{4}$ and $\mathrm{Ni}$ in reactive wetting of eutectic $\mathrm{SnPb}$ on $\mathrm{Ni}$, Journal of Applied Physics 109 (2011) 123513.

[77] W. Jeitschko, B. Jaberg, Structure refinement of $\mathrm{Ni}_{3} \mathrm{Sn}_{4}$, Acta Crystallographica Section B: Structural Crystallography and Crystal Chemistry 38 (1982) 598-600.

[78] C. Fairhurst, J. Cohen, The crystal structures of two compounds found in dental amalgam: $\mathrm{Ag}_{2} \mathrm{Hg}_{3}$ and $\mathrm{Ag}_{3} \mathrm{Sn}$, Acta Crystallographica Section B: Structural Crystallography and Crystal 
Chemistry 28 (1972) 371-378. 


\section{FIGURE CAPTION}

Fig. 1. (Color online) Schematic illustrations showing the 2D atomic site correspondence or heteroepitaxial relationship at the interface between (a) $\eta-\mathrm{Cu}_{6} \mathrm{Sn}_{5}$ and (001) $\mathrm{Cu}$. (b) $\eta-\mathrm{Cu}_{6} \mathrm{Sn}_{5}$ and (111) $\mathrm{Cu}$. The shaded area in the hexagonal lattice $\eta-\mathrm{Cu}_{6} \mathrm{Sn}_{5}$ shows the lattice plane in parallel with the $\mathrm{Cu}$ surface. The circles of dash line in (b) represent the atomic sites that are not in the same atomic layer with the other atoms.

Fig. 2. (Color online) Plots showing the chemical free energy of $\mathrm{Cu}(\alpha)$, liquid $\mathrm{Sn}(\mathrm{L})$, and $\eta-\mathrm{Cu}_{6} \mathrm{Sn}_{5}$ as a function of composition at $523 \mathrm{~K}$.

Fig. 3. Schematic drawing showing the simulation supercell with initial distribution of the $\mathrm{Cu}$ substrate and liquid Sn.

Fig. 4. (Color online) Time-evolution of the stress fields of (a) $\sigma_{11}$, (b) $\sigma_{22}$, and (c) $\sigma_{11}+\sigma_{22}$, with the dash lines outlining the growth of $\eta-\mathrm{Cu}_{6} \mathrm{Sn}_{5}$ (blue for variant 1 and orange for variant 2) on $(001) \mathrm{Cu}$. The stress fields are calculated based on the $\mathrm{L}_{1}$ coordinate.

Fig. 5. (Color online) Time-evolution of the stress field distributions of (a) $\sigma_{11}$, (b) $\sigma_{22}$, and (c) $\sigma_{11}+$ $\sigma_{22}$ with the dash lines outlining the growth $\eta-\mathrm{Cu}_{6} \mathrm{Sn}_{5}$ (blue for variant 1 , orange for variant 2 and red for variant 3) on (111) $\mathrm{Cu}$. The stress fields are calculated based on the $\mathrm{L}_{2}$ coordinate.

Fig. 6. (a)-(e) 3D phase-field simulation results showing the time-evolution of the heteroepitaxial growth of $\eta-\mathrm{Cu}_{6} \mathrm{Sn}_{5}$ on a (001) $\mathrm{Cu}$ substrate. (f) Top-view of a scanning electron 
microscope (SEM) image showing the pattern and morphology of $\eta-\mathrm{Cu}_{6} \mathrm{Sn}_{5}$ on a (001) $\mathrm{Cu}$ substrate. (Courtesy of Prof. K. N. Tu [10])

Fig. 7. (Color online) 3D phase-field simulation result (same as what shown in Figure 6(e)) showing spatial distribution of the two $\eta-\mathrm{Cu}_{6} \mathrm{Sn}_{5}$ variants (represented by two different colors) on (001) $\mathrm{Cu}$.

Fig. 8. (a)-(e) $3 \mathrm{D}$ phase-field simulation results showing heteroepitaxial growth of $\eta-\mathrm{Cu}_{6} \mathrm{Sn}_{5}$ on a (111) Cu substrate. (f) Top-view of a scanning electron microscope (SEM) image showing the pattern and morphology of $\eta-\mathrm{Cu}_{6} \mathrm{Sn}_{5}$ on a (111) $\mathrm{Cu}$ substrate. (Courtesy of Prof. Z. F. Zhang [22])

Fig. 9. (Color online) 3D simulation result (the same as what shown in Figure 8(e)) showing spatial distribution of the three $\eta-\mathrm{Cu}_{6} \mathrm{Sn}_{5}$ variants (represented by three different colors) on (111) $\mathrm{Cu}$.

Fig. 10. (a)-(e) 3D phase-field simulation results showing growth of $\eta-\mathrm{Cu}_{6} \mathrm{Sn}_{5}$ on a $\mathrm{Cu}$ substrate without heteroepitaxial constraints. (f) Top-view of a scanning electron microscope (SEM) image showing the scalloped grains of $\eta-\mathrm{Cu}_{6} \mathrm{Sn}_{5}$ on a polycrystalline substrate. (Courtesy of Prof. Z. F. Zhang [22])

Fig. 11. (Color online) Polar plots of $B_{p p}(\mathbf{n})$ showing the density of coherency elastic strain energy of each $\eta-\mathrm{Cu}_{6} \mathrm{Sn}_{5}$ variant projected on (a) (001) plane and (b) (111) plane of $\mathrm{Cu}$. The calculations of (a) and (b) are based on the $\mathrm{L}_{1}$ and $\mathrm{L}_{2}$ coordinates, respectively. The arrows indicate the habit plane normal direction of each variant. 
(a)

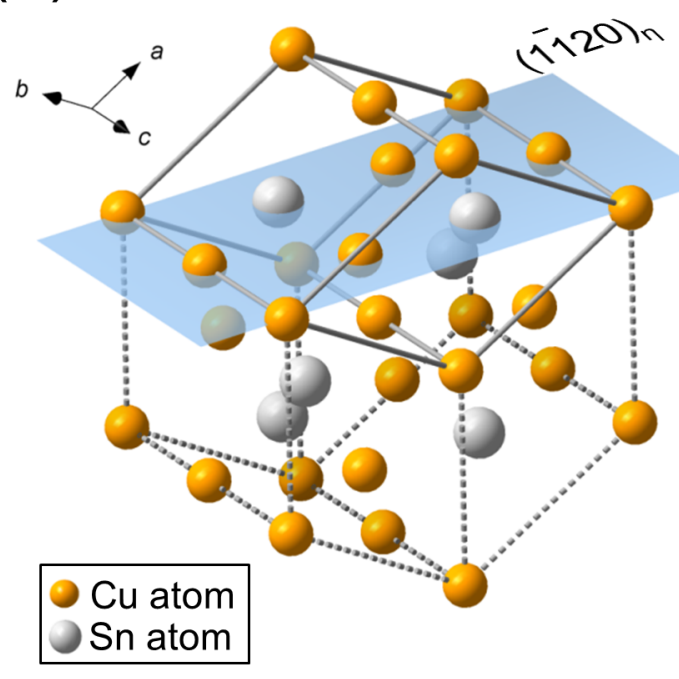

(b)

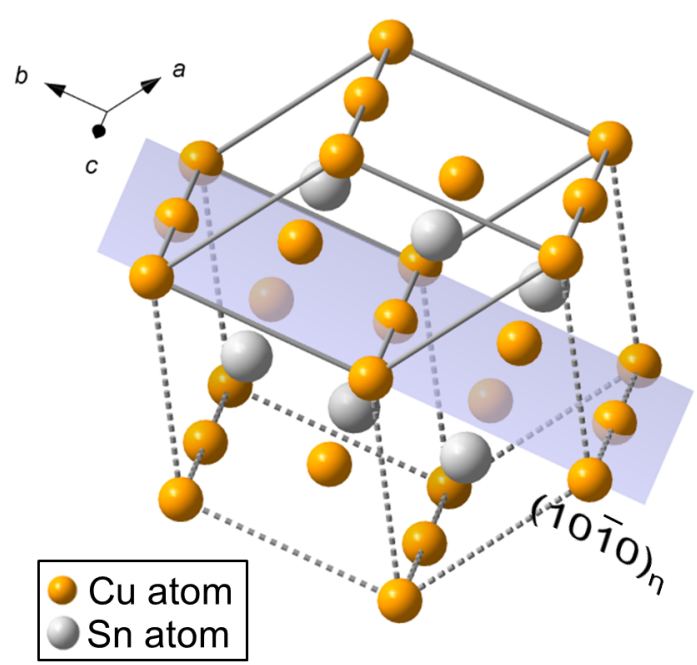
fig1
$[110]_{\mathrm{Cu}} \mathrm{Cu}:(001)$ plane

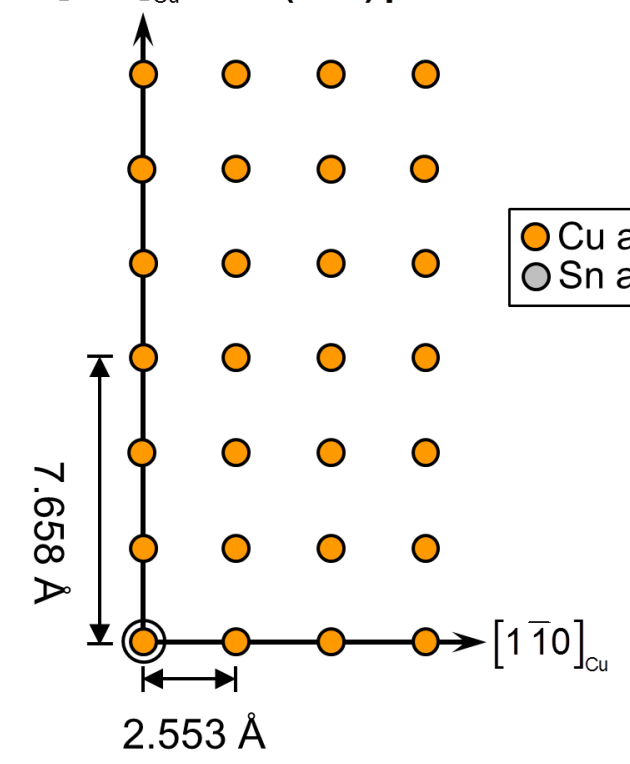

$[\overline{1} 100]_{\eta} \eta-\mathrm{Cu}_{6} \mathrm{Sn}_{5}:(11 \overline{2} \mathbf{0})$ plane

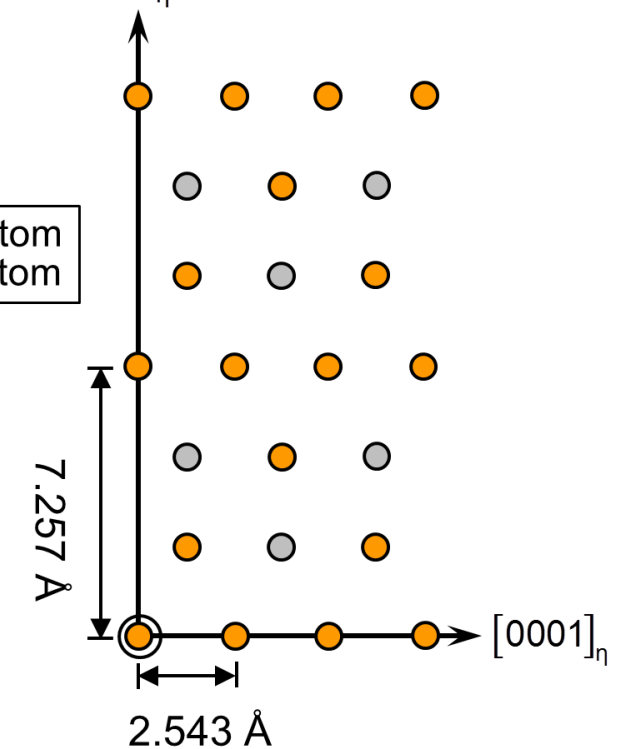

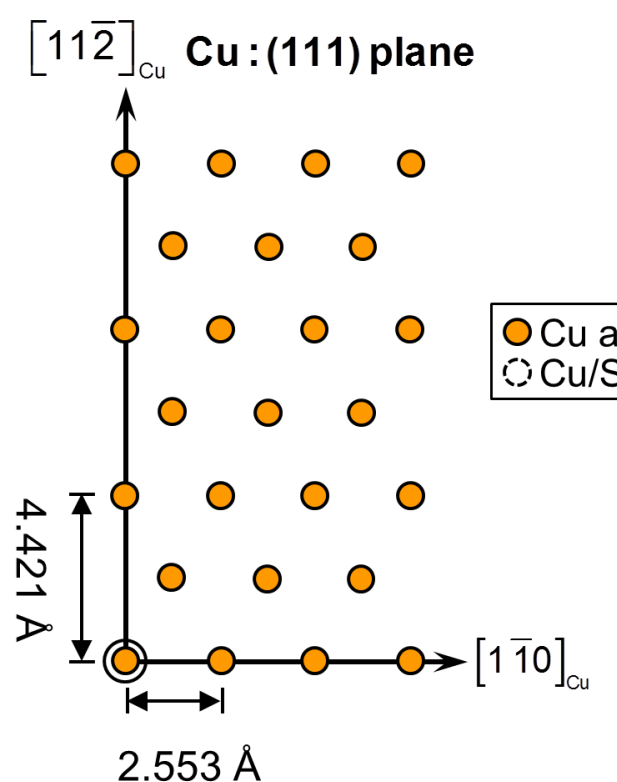

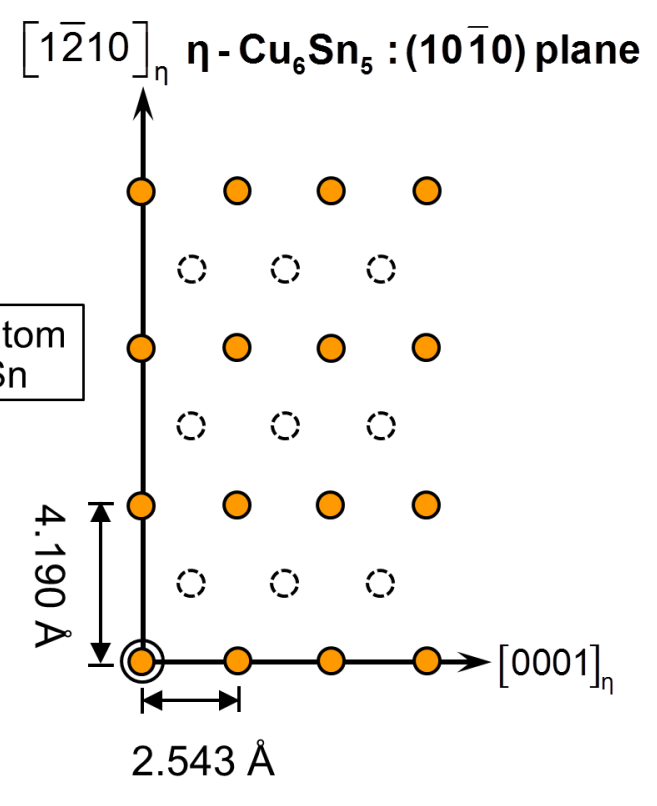




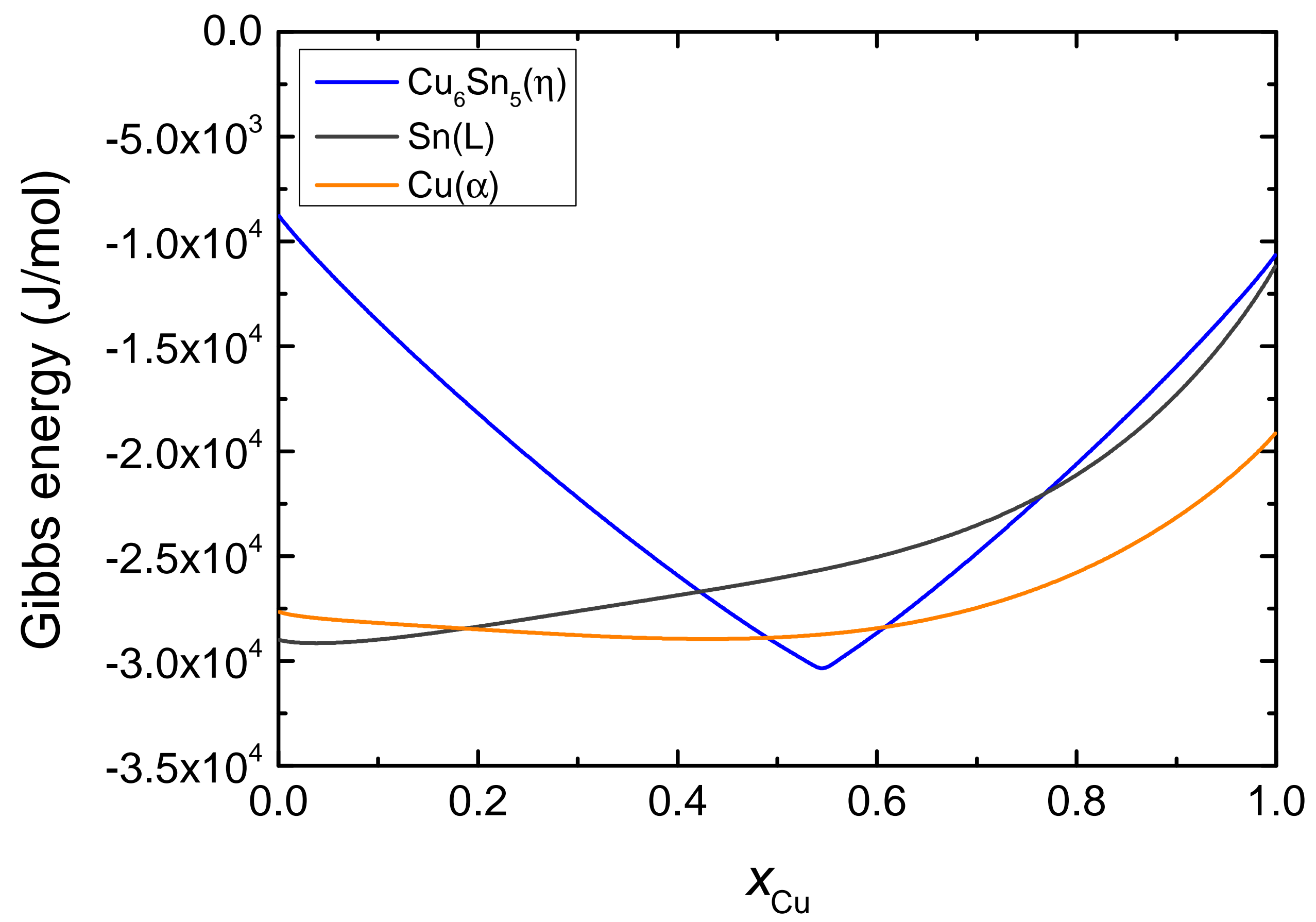


fig3

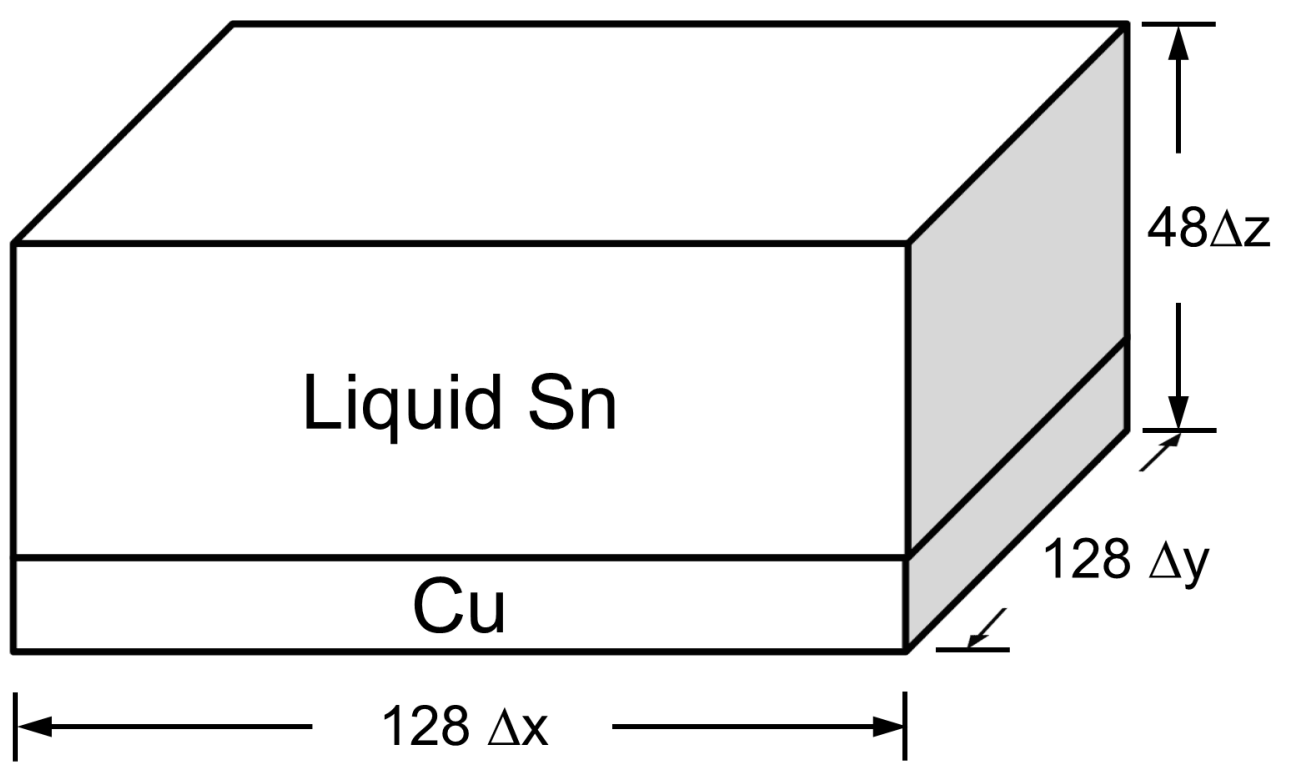


(a) $\sigma_{11}$

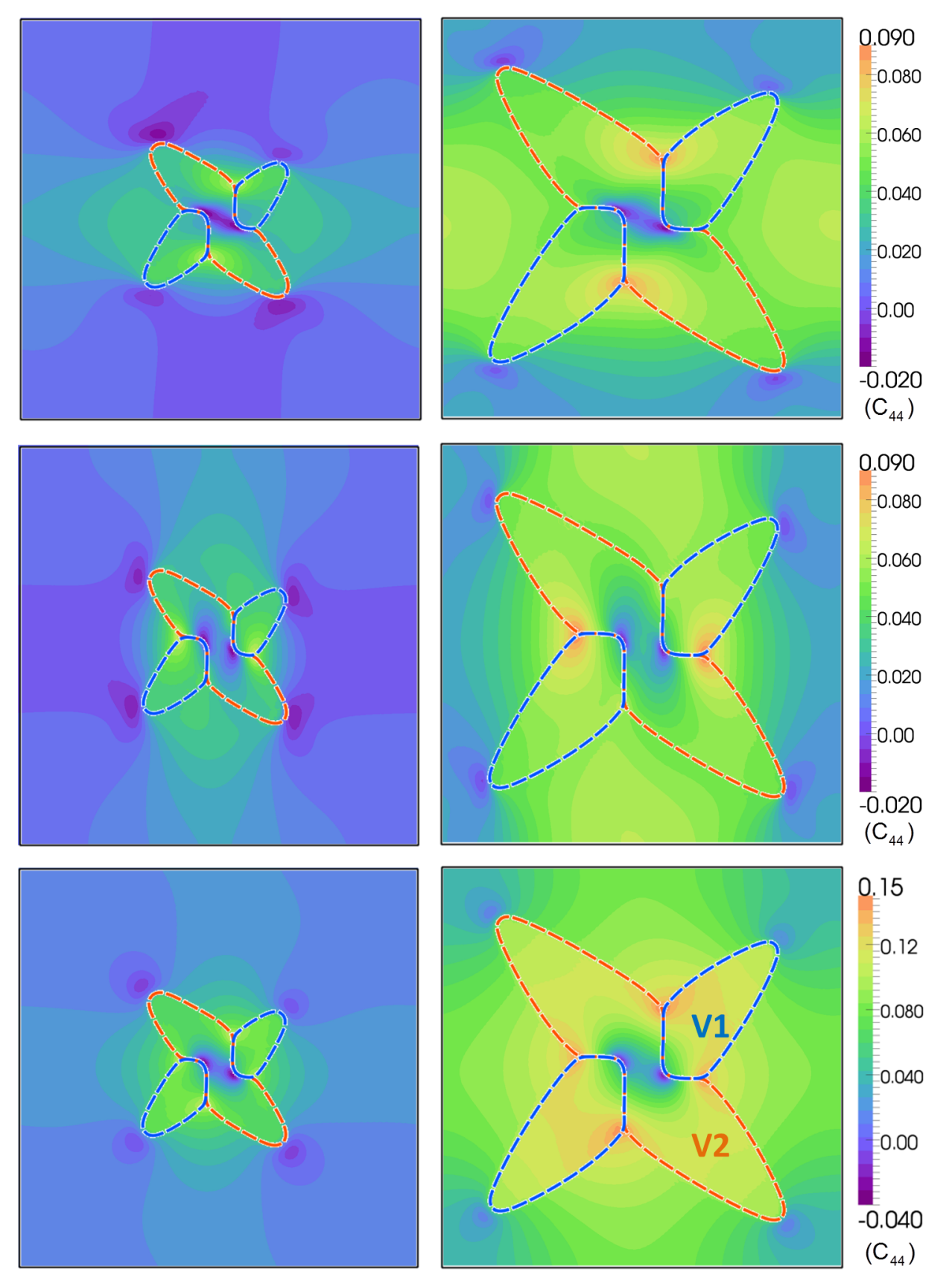


(a) $\sigma_{11}$
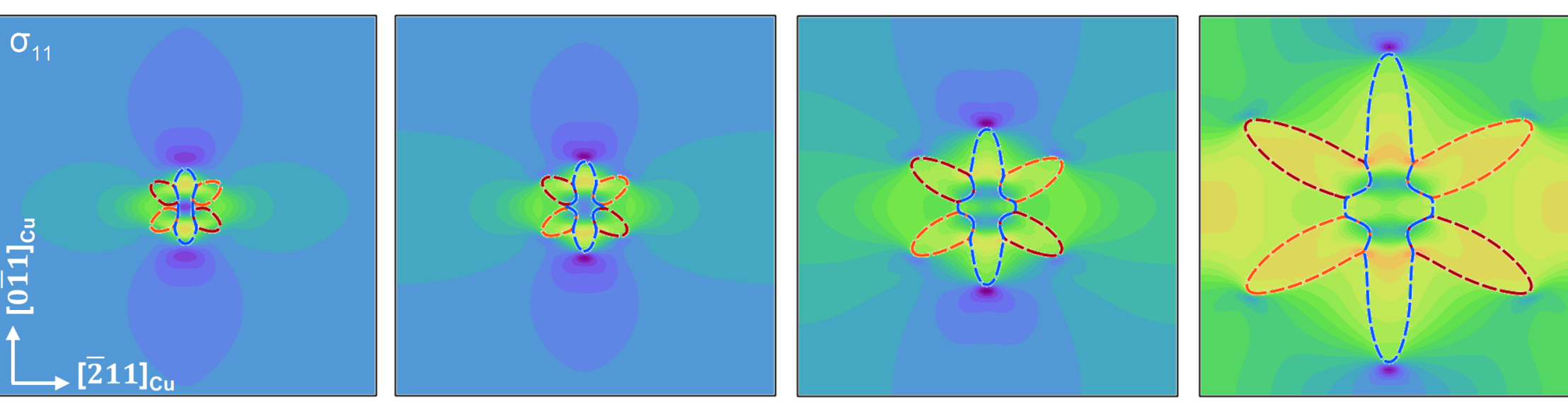

(b)
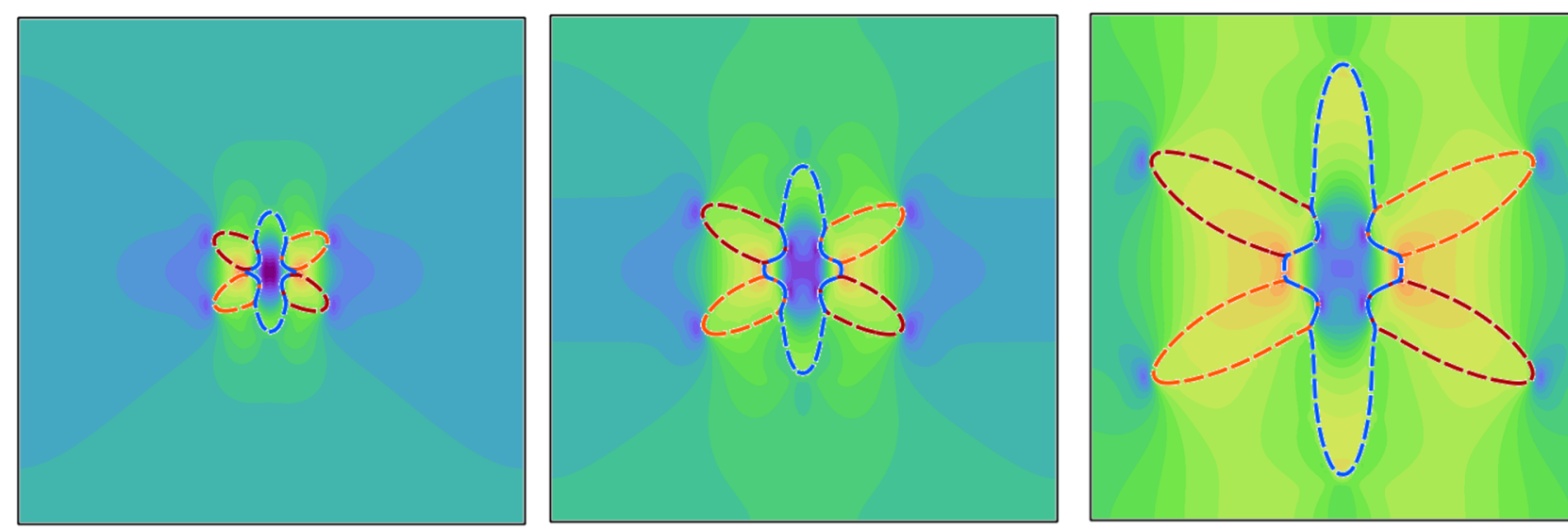

$\left(\mathrm{C}_{44}\right)$

$[\overline{2} 11]_{\mathrm{Cu}}$

(c) $\sigma_{11}+\sigma_{22}$
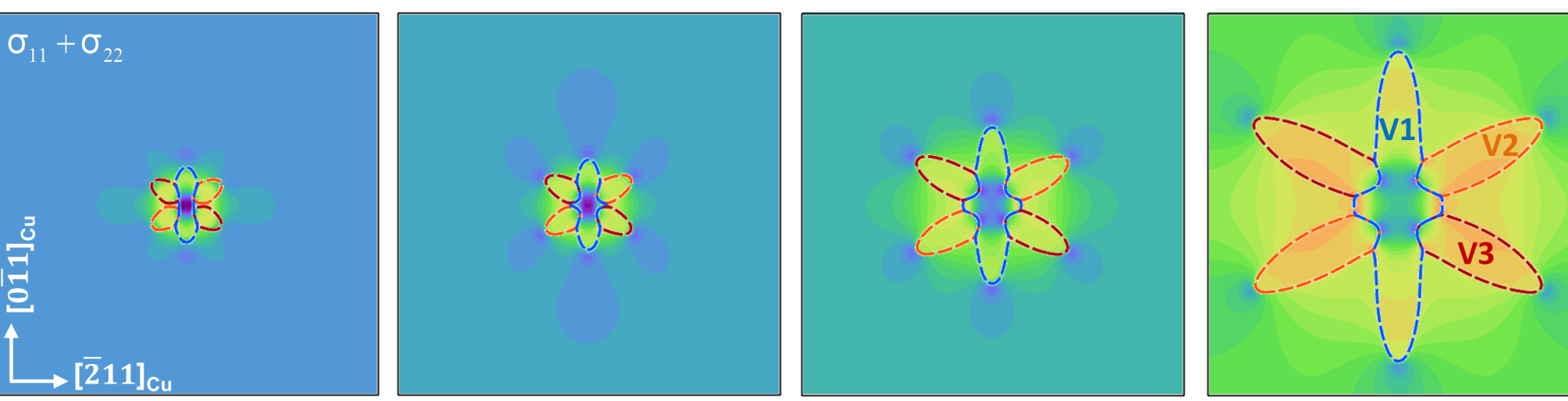

0.070
0.060
0.040
0.020
0.00
0.020
0.040
$\left(C_{44}\right)$

0.12

0.080

0.040

0.00

$-0.040$

$-0.060$

$\left(\mathrm{C}_{44}\right)$ 

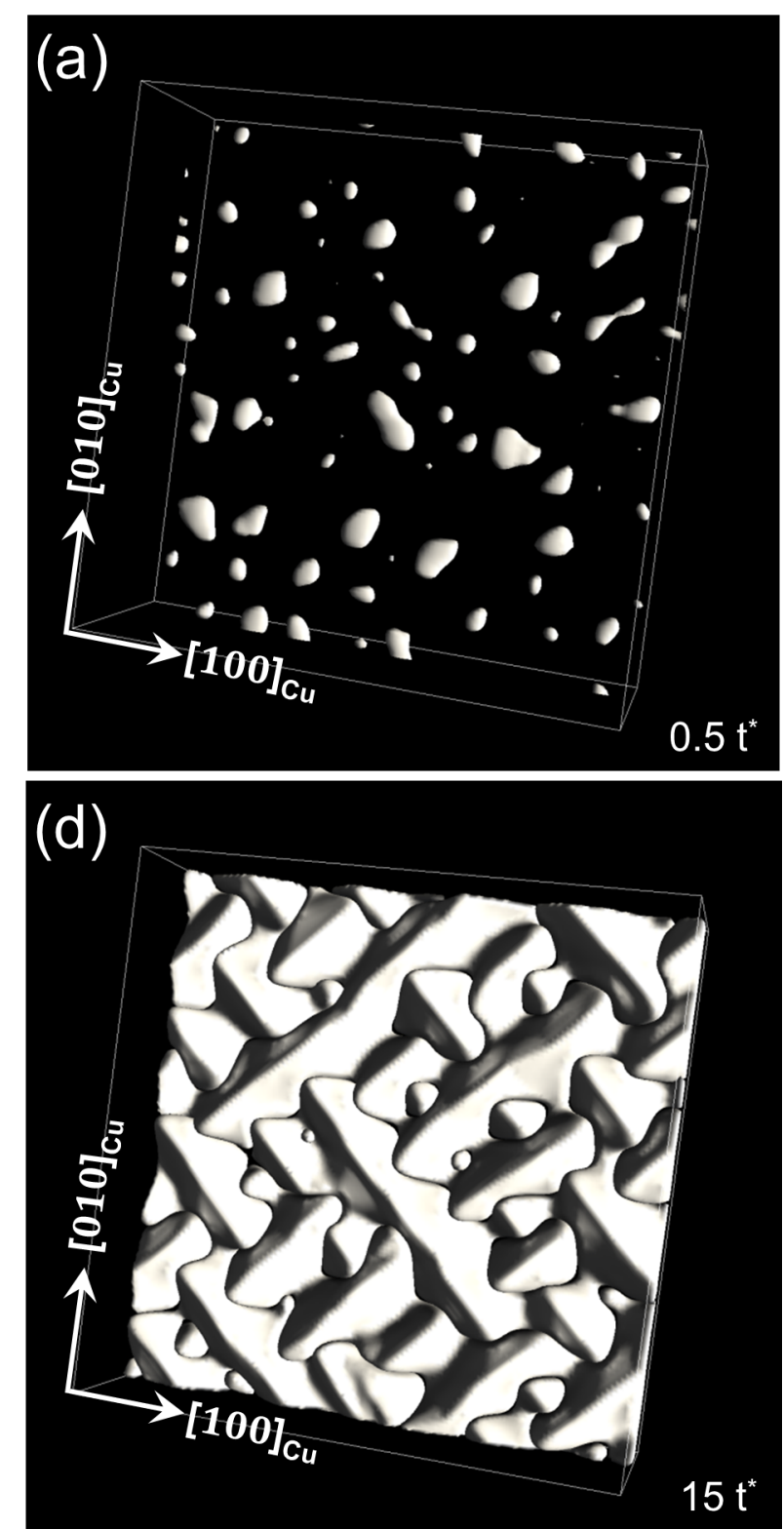
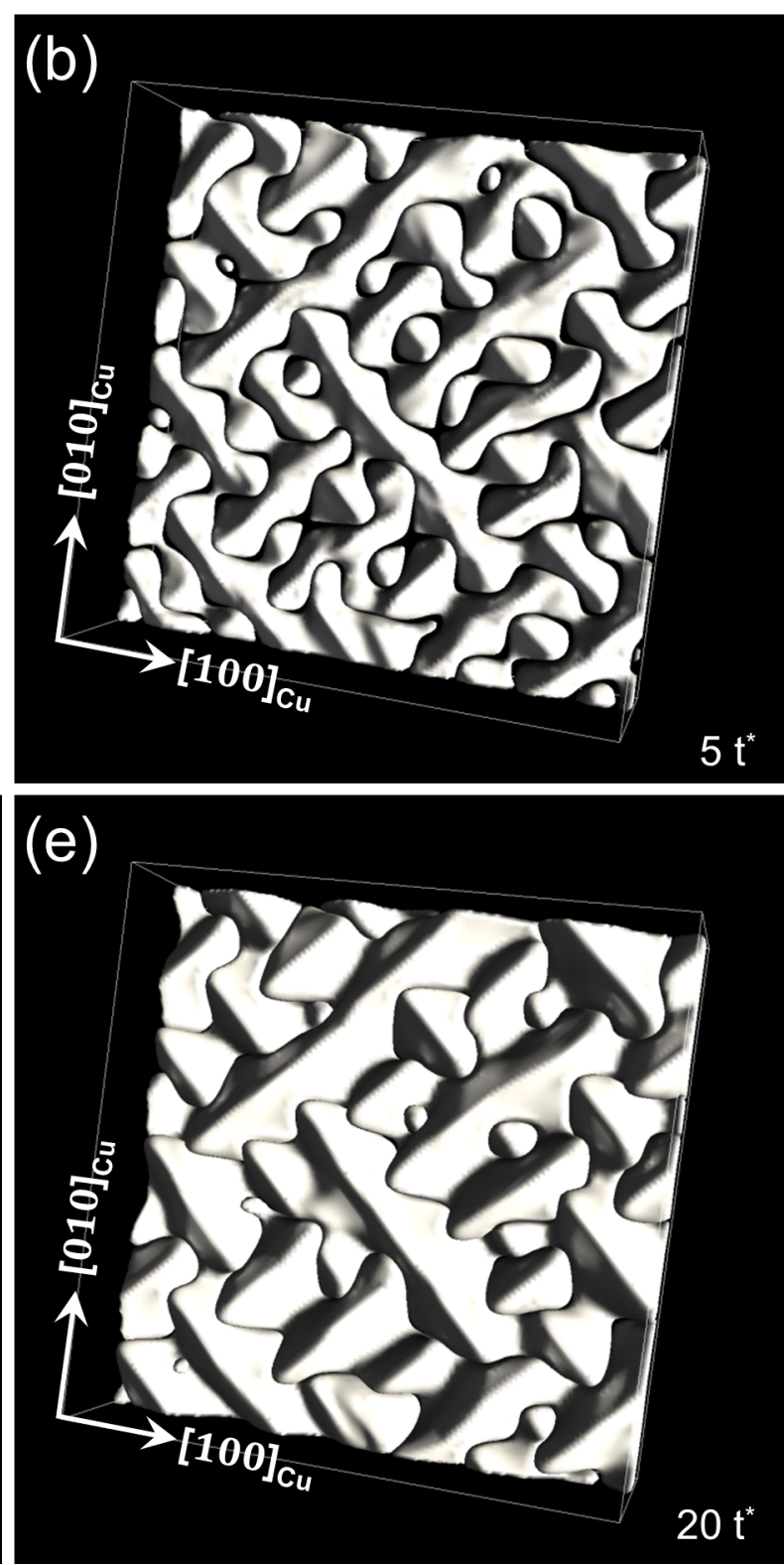

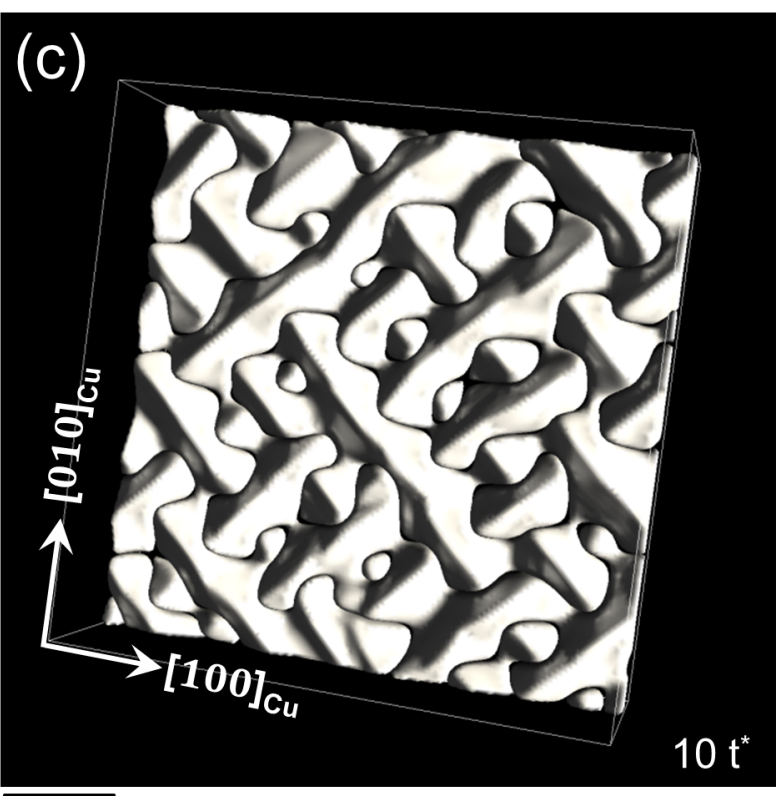

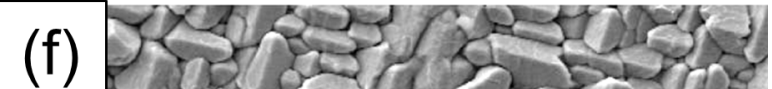

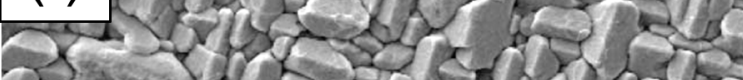

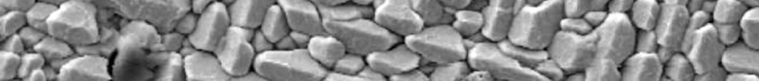

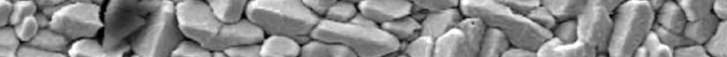
C.

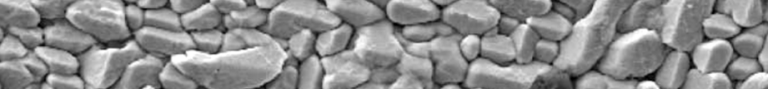

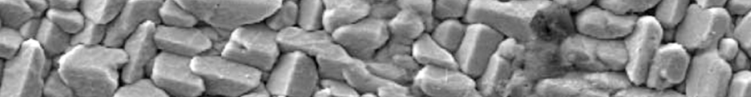

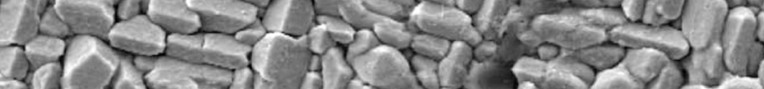
30.67

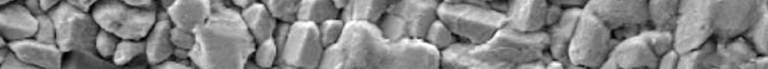

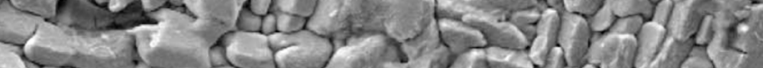

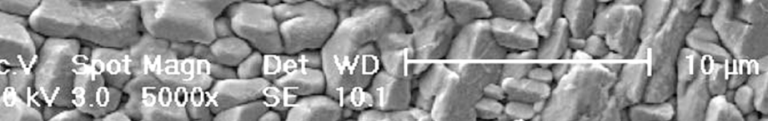


fig 7

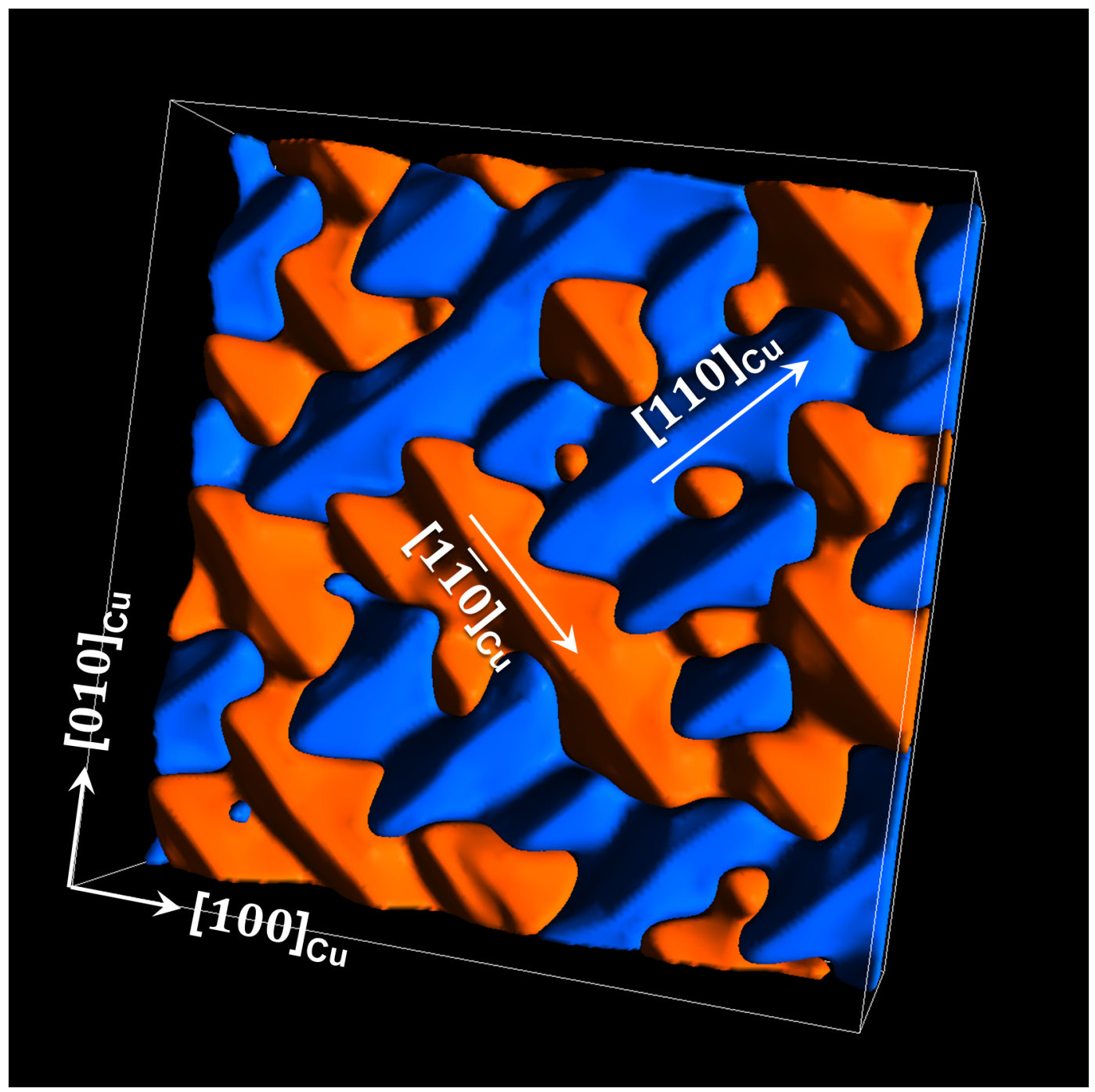



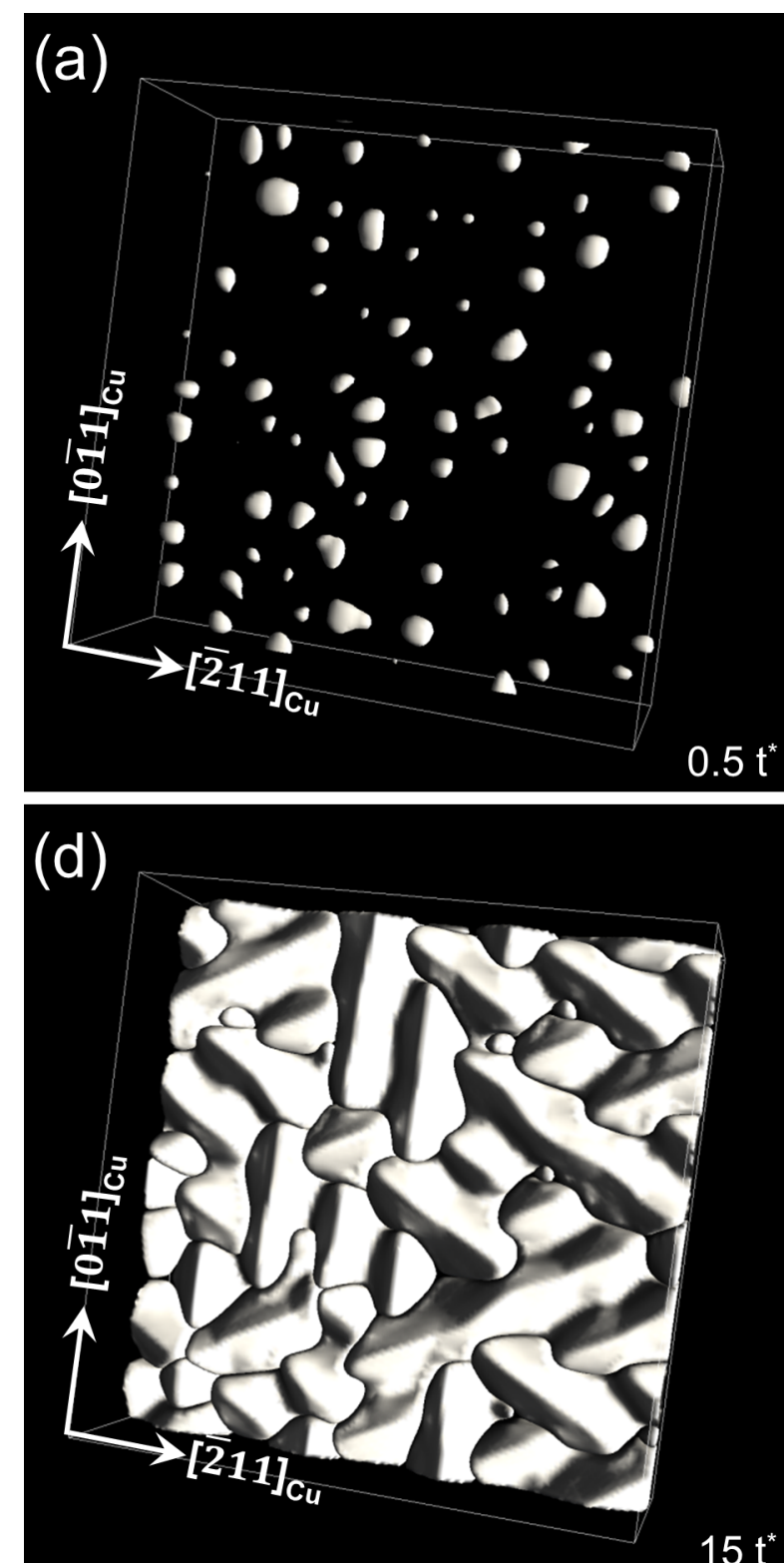
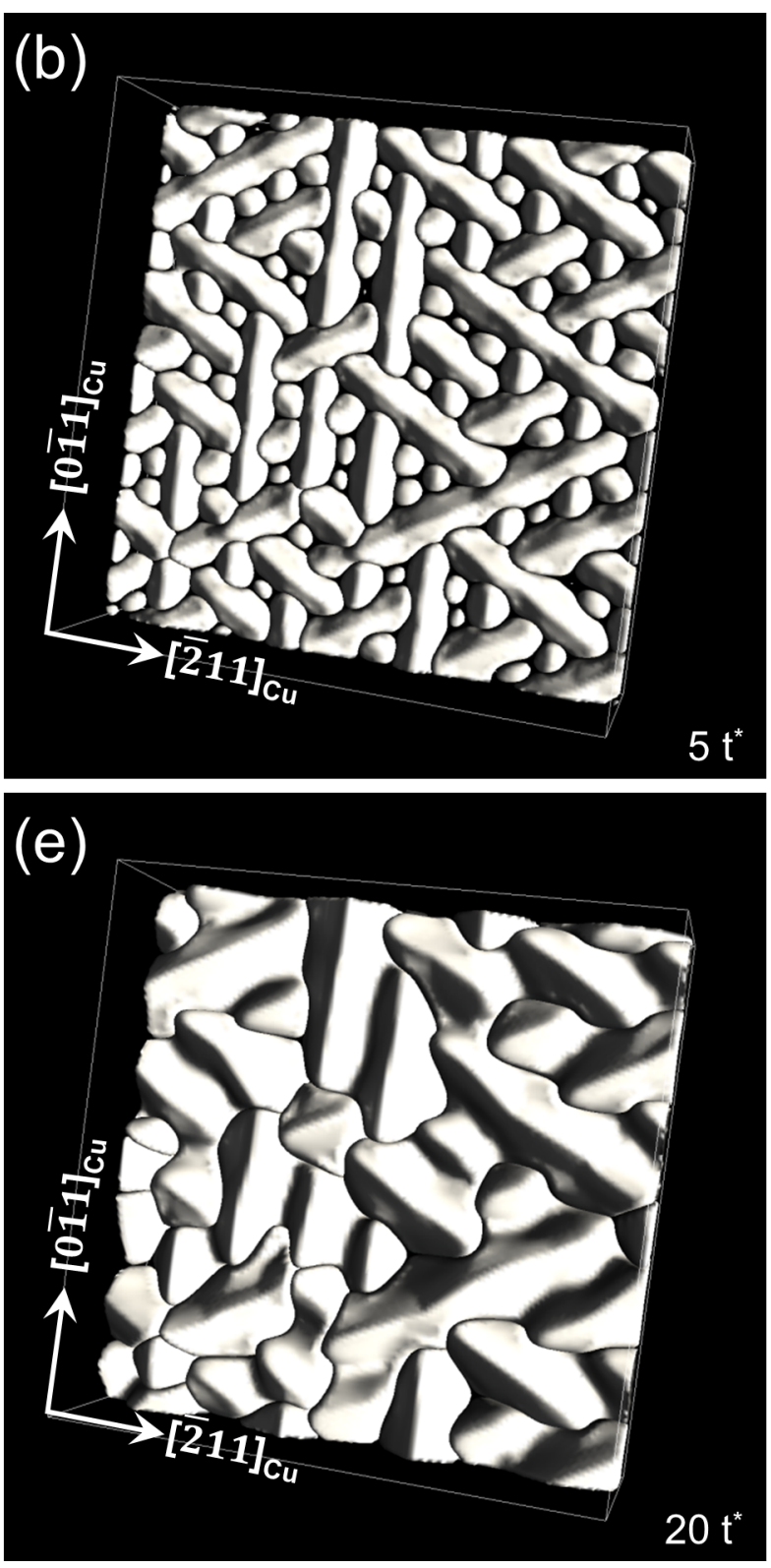
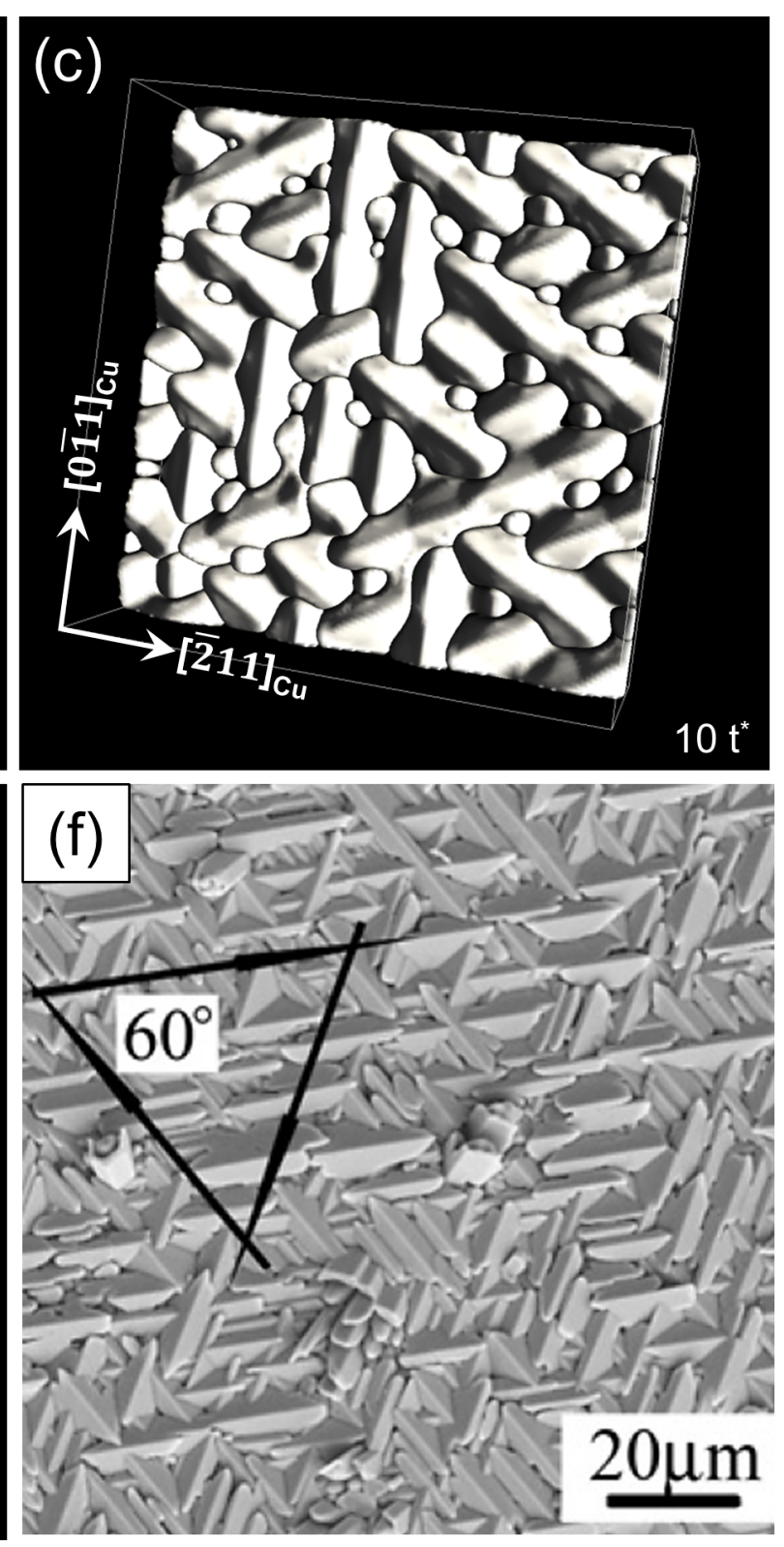
fig 9

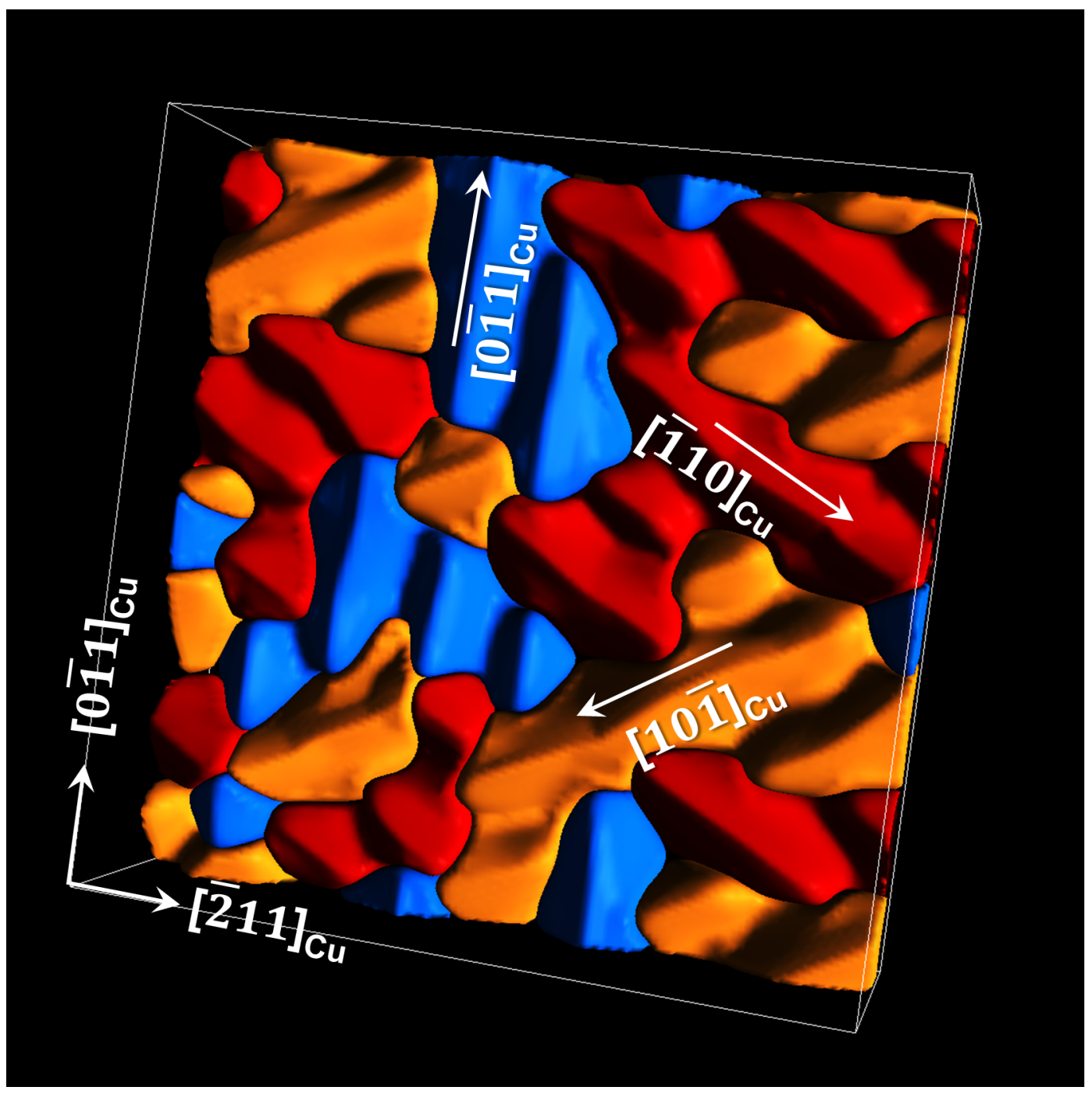



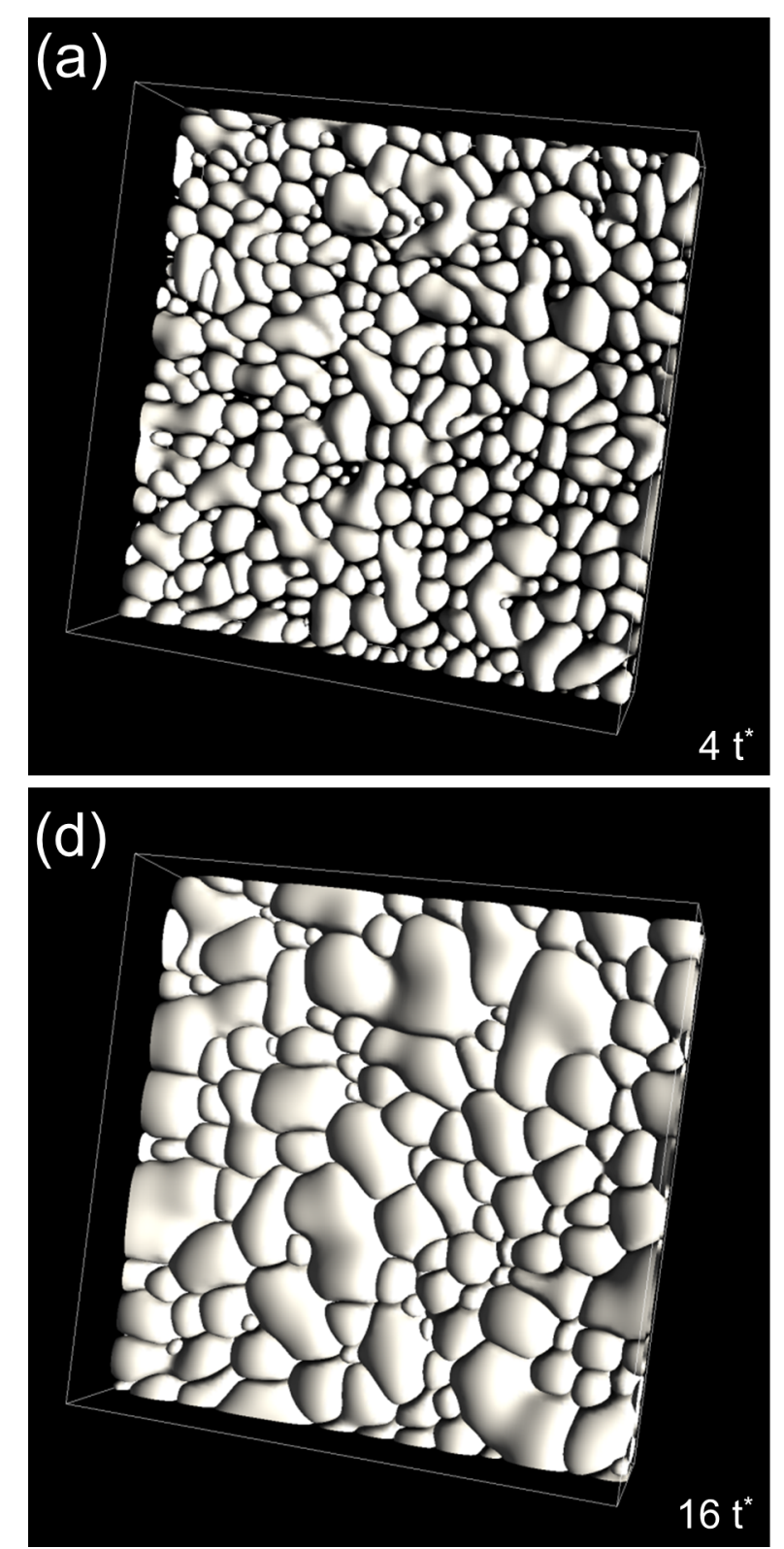
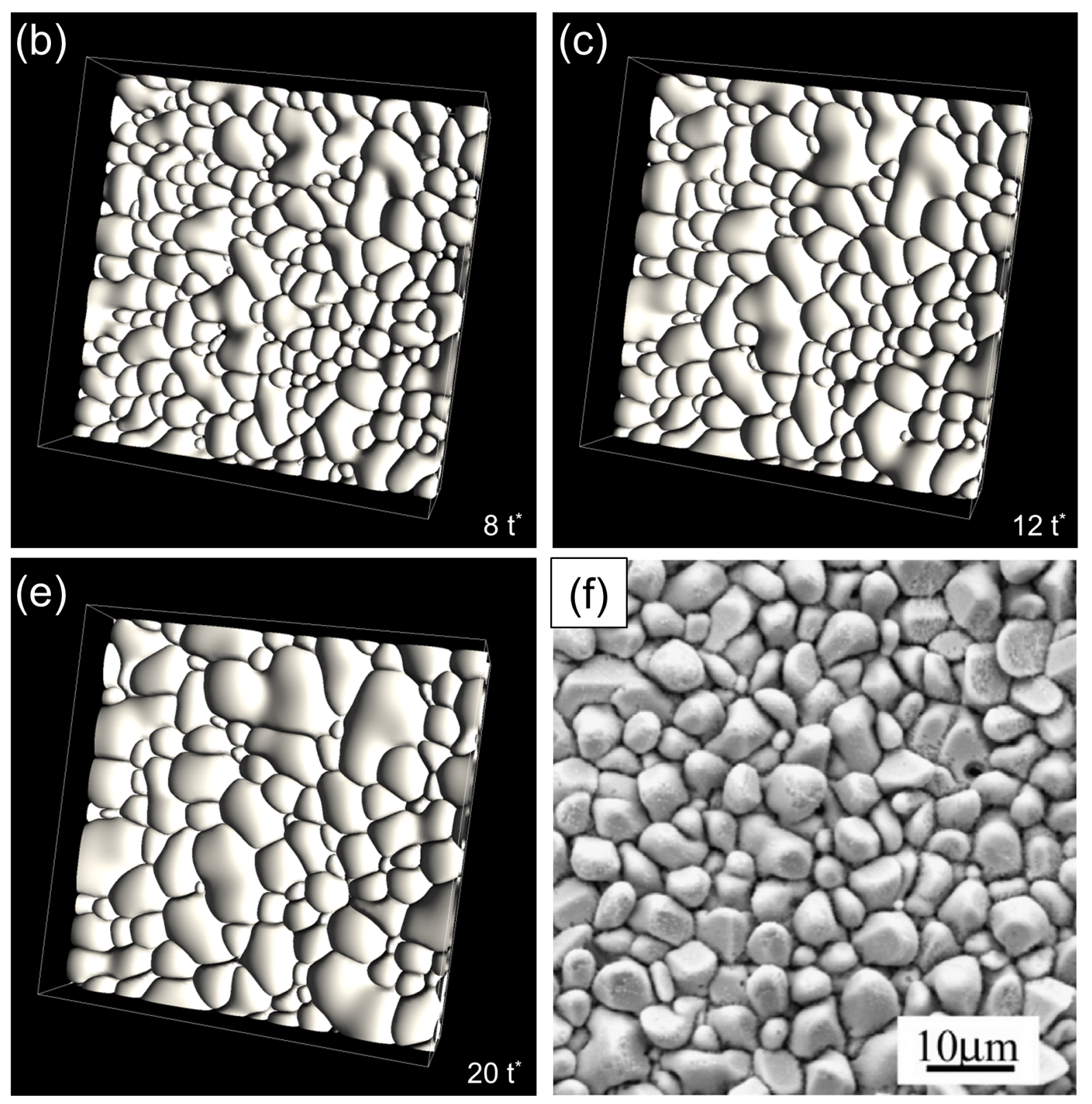

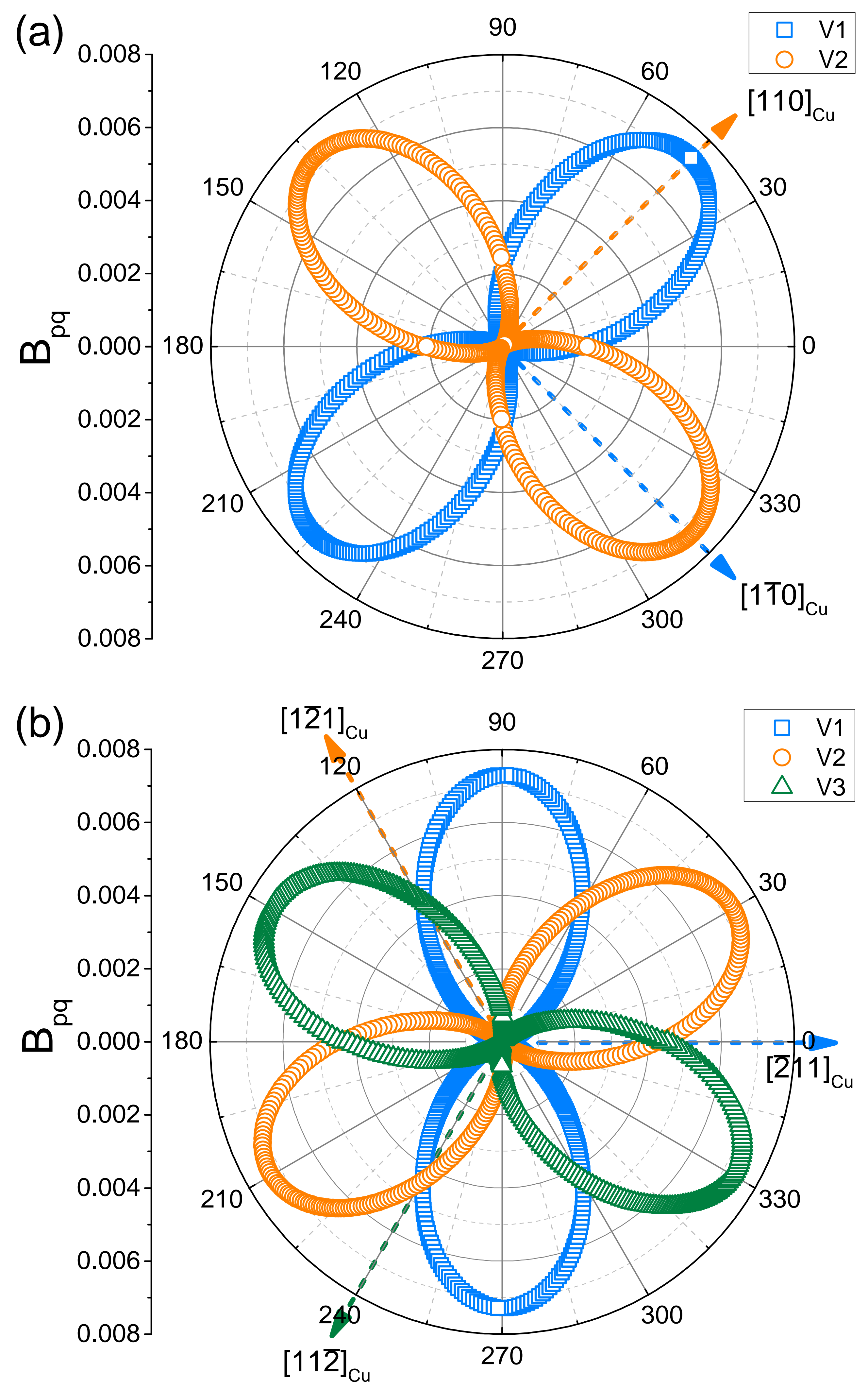

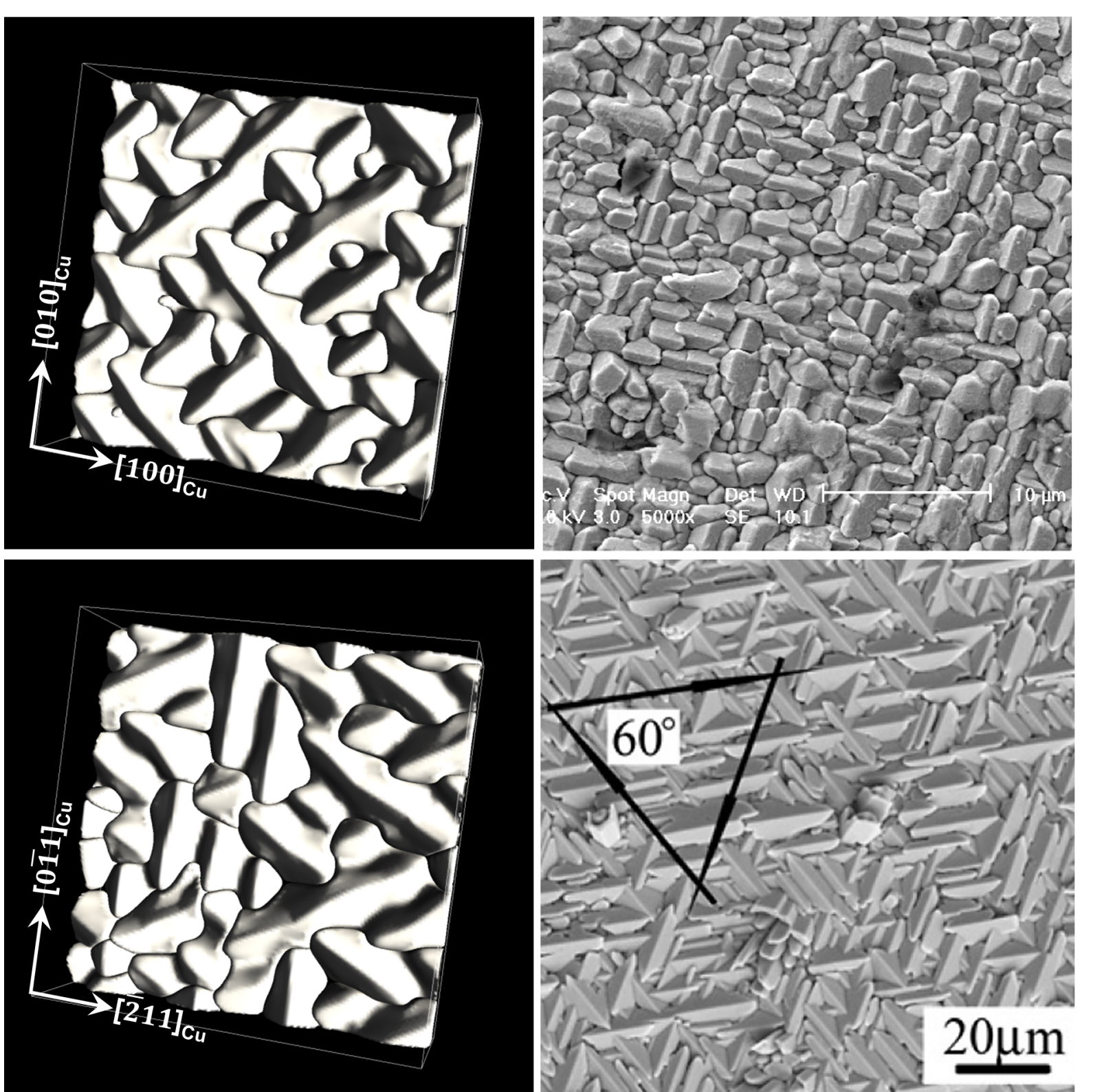